\title{
Maresin 1 activates LGR6 receptor promoting phagocyte immunoresolvent functions
}

\author{
Nan Chiang, Stephania Libreros, Paul C. Norris, Xavier de la Rosa, and Charles N. Serhan \\ Center for Experimental Therapeutics and Reperfusion Injury, Department of Anesthesiology, Perioperative and Pain Medicine, Brigham and Women's Hospital and Harvard Medical School, Boston, \\ Massachusetts, USA.
}

\begin{abstract}
Resolution of acute inflammation is an active process orchestrated by endogenous mediators and mechanisms pivotal in host defense and homeostasis. The macrophage mediator in resolving inflammation, maresin 1 (MaR1), is a potent immunoresolvent, stimulating resolution of acute inflammation and organ protection. Using an unbiased screening of greater than 200 GPCRs, we identified MaR1 as a stereoselective activator for human leucine-rich repeat containing G protein-coupled receptor 6 (LCR6), expressed in phagocytes. MaR1 specificity for recombinant human LGR6 activation was established using reporter cells expressing LCR6 and functional impedance sensing. MaR1-specific binding to LCR6 was confirmed using ${ }^{3} \mathrm{H}$-labeled MaR1. With human and mouse phagocytes, MaR1 (0.01-10 nM) enhanced phagocytosis, efferocytosis, and phosphorylation of a panel of proteins including the ERK and cAMP response element-binding protein. These MaR1 actions were significantly amplified with LGR6 overexpression and diminished by gene silencing in phagocytes. Thus, we provide evidence for MaR1 as an endogenous activator of human LGR6 and a novel role of LGR6 in stimulating MaR1's key proresolving functions of phagocytes.
\end{abstract}

\section{Introduction}

The acute inflammatory response is a protective mechanism to injury or microbial invasion and ideally is self-limited, leading to complete resolution (1). The resolution of inflammation is temporally and spatially governed by cellular and molecular events as well as endogenous mediators, leading to clearance of inflammatory infiltrates, restoration of tissue integrity, and return to function (2). When excessive or unresolved, this protective response progresses to chronic inflammation, which is a unifying component in many organ-specific diseases and can be viewed as failed resolution (2). Phagocytes play pivotal roles in both host defense and in resolution of inflammation (3). Neutrophils (PMN), for example, when recruited in excess or unable to exit the site of inflammation, can cause unintended collateral tissue damage that amplifies ongoing inflammation (4). As many as $60 \%$ of Americans have at least 1 chronic disease or condition, such as heart disease, stroke, chronic lung disease, cancer, diabetes, arthritis, and Alzheimer's disease, which are significant burdens in health and economic costs (5). Understanding the endogenous resolution mechanisms such as proresolving lipid mediators (LMs), peptides, and gaseous mediators (6-9) in inflammation are therefore urgently needed to identify new potential therapeutic targets.

The specialized proresolving mediators (SPMs) are endogenous families of chemical mediators derived from polyunsaturat-

Conflict of interest: The authors have declared that no conflict of interest exists. Copyright: () 2019, American Society for Clinical Investigation.

Submitted: April 8, 2019; Accepted: August 28, 2019; Published: October 28, 2019

Reference information: / Clin Invest. 2019;129(12):5294-5311.

https://doi.org/10.1172/JCl129448. ed fatty acids including arachidonic acid (AA), eicosapentaenoic acid (EPA), and docosahexaenoic acid (DHA) and collectively constitute a superfamily of mediators in inflammatory exudates $(2,7)$. SPMs are temporally biosynthesized in inflammatory exudates to control inflammation via stimulating resolution programs without immunosuppression and are organ protective. Among them, a macrophage mediator in resolving inflammation, denoted maresin 1 (MaR1) was first identified in human macrophages (MФ) (10). Human MФ carry out 14-lipoxygenation of DHA with molecular oxygen to produce a 14S-hydroperoxy-containing intermediate that is enzymatically transformed to a unique intermediate 13S,14S-epoxy-maresin (eMaR; 13S,14S-epoxyDHA: 13S,14S-epoxy-docosa-4Z,7Z,9E,11E,16Z,19Z-hexaenoic acid), followed by enzymatic hydrolysis to produce MaR1 (10, $11)$. The complete stereochemistry of MaR1 is $7 R, 14 S$-dihydroxydocosa-4Z,8E,10E,12Z,16Z,19Z-hexaenoic acid (12), and its total organic synthesis was achieved and confirmed by several independent teams (12-14).

MaR1 is produced in humans $(7,15-19)$, and its defining physiologic functions include limiting $\mathrm{PMN}$ infiltration in murine peritonitis, enhancing human $\mathrm{M} \Phi$ uptake of apoptotic PMNs (16), the hallmarks of proresolving mechanisms. By controlling inflammation after surgical tissue injury, MaR1 accelerates planaria regeneration (12). MaR1 is neuroprotective, reduces pain, and is organ protective in experimental diseases in vivo (12, 20, 21 and reviewed in ref. 7). Hence, via its distinct actions on PMN and M, MaR1 is a key SPM in a wide range of tissues, such as adipose, lymphoid, brain, and nervous tissues (16, 19, 20, 22). Herein, we screened orphan and known receptors and report that a GPCR, leucine-rich repeat- containing G protein-coupled receptor 6 (LGR6) is activated by MaR1. LGR6 is present in mul- 
tiple tissues and is known to promote repair and regeneration (23). By using overexpression and/or knockdown of LGR6, we uncovered a new role for LGR6 in mediating MaR1's proresolving actions with human and mouse phagocytes.

\section{Results}

MaR1 receptor activity and expression. LMs such as eicosanoids and resolvins exhibit potent stereoselective actions via activating cell surface receptors, which belong to the GPCR superfamily, that amplify intracellular signals within minutes following ligand binding to regulate a variety of cellular functions $(7,24)$. Because MaR1 exhibits rapid (seconds to minutes) and potent (picomolar to nanomolar) stereoselective actions with human cells, we questioned whether there are specific cell surface GPCRs mediating its actions. To address this theory, we employed an unbiased screening system using GPCR and $\beta$-arrestin coupling (see Methods) to monitor MaR1-receptor interactions (Figure 1). The advantage of this system is that it does not rely on activation of classic intracellular second messengers, such as intracellular $\left[\mathrm{Ca}^{2+}\right]$ (25). Among 77 orphan human GPCRs, 2 receptors gave the strongest responses increasing chemiluminescence (RLU) with MaR1 (10 nM), namely LGR6 and GPR148 (Figure 1A). These 2 receptors were selected for further investigation based on the criteria that the mean RLU in the presence of MaR1 was larger than the mean + 2 SD obtained with vehicle alone (Supplemental Table 1; supplemental material available online with this article; https:/doi. org/10.1172/JCI129448DS1). To rule out false-positive results, we further evaluated human LGR6 and GPR148 using this $\beta$ arrestin-based ligand receptor interaction system. MaR1 $\left(10^{-13}\right.$ $\mathrm{M}-10^{-8} \mathrm{M}$ ) did not significantly increase RLU in GPR148 cells (Figure 1B). In comparison, MaR1 and MaR1 carboxymethyl ester (ME) $\left(10^{-13} \mathrm{M}-10^{-8} \mathrm{M}\right)$ each dose-dependently increased these signals with LGR6-expressing cells (Figure 1, B and C).

Next, we examined pathway specificity for human recombinant LGR6 using the CHO- $\beta$-arrestin-LGR6 cells and evaluated cysteinyl-containing maresins, namely maresin conjugates in tissue regeneration (MCTR), the potent bioactive family members MCTR1, MCTR2, and MCTR3 that directly control tissue regeneration (7). (See Figure $1 \mathrm{H}$ for the maresin family biosynthesis.) Unlike MaR1, neither of the MCTR at equimolar concentrations $\left(10^{-13} \mathrm{M}-10^{-8} \mathrm{M}\right)$ significantly activated LGR6 (Figure 1D). Rspondins, the Wnt pathway agonists, can bind to the LGR receptor family (26). Among them, R-spondin-2 (Rspo-2) is the most potent ligand reported for LGR6 (27). Hence, we tested Rspo-2, which at the same concentration ranges did not significantly activate $\mathrm{CHO}$ $\beta$-arrestin-LGR6 cells. In addition, Rspo-2, when added together with MaR1, significantly reduced MaR1-initiated activation of these cells (Figure 1E). To investigate whether temperature could affect MaR1 activation of LGR6, we examined $\beta$-arrestin activity at $4^{\circ} \mathrm{C}, 25^{\circ} \mathrm{C}, 37^{\circ} \mathrm{C}$, and $40^{\circ} \mathrm{C}$. MaR1 $(0.1-10 \mathrm{nM})$ significantly activated LGR6 at $37^{\circ} \mathrm{C}$ and $40^{\circ} \mathrm{C}$, but apparently not at $25^{\circ} \mathrm{C}$ and $4^{\circ} \mathrm{C}$ (Figure $1 \mathrm{~F}$ and Supplemental Figure $1 \mathrm{~A}$ ). We also evaluated $\beta$ arrestin activity at $\mathrm{pH} 6.5,7.5$, and 8.5 and found that activation of LGR6 by 0.1-nM MaR1 was significantly higher at $\mathrm{pH} 8.5$ than at pH 6.5 (Supplemental Figure 1B). These results indicate that in addition to MaR1 concentrations, both temperature and $\mathrm{pH}$ are likely to affect MaR1 interactions with LGR6.
Because cAMP, a second messenger following GPCR activation, plays an essential role in macrophage functions and phenotypes $(28,29)$, we determined whether MaR1 regulated cAMP with recombinant human LGR6. MaR1 at 10 to $100 \mathrm{nM}$ significantly increased intracellular cAMP accumulation with LGR6-transfected human embryonic kidney (HEK)-293 cells, a response that was not apparent in mock-transfected HEK-293 cells (Figure 1G). These results demonstrated that MaR1 is a selective ligand activating human recombinant LGR6 and evoking second-messenger cAMP.

We also screened a panel of known GPCRs, containing 158 receptors. MaR1 $(10 \mathrm{nM})$ did not appear to activate receptors for prostaglandins (PTGER2, PTGER3, PTGER4, PTGIR), leukotriene $\mathrm{B}_{4}\left(\mathrm{LTB}_{4}\right)\left(\mathrm{LTB}_{4} \mathrm{R} / \mathrm{BLT} 1\right)$, and thromboxane $\mathrm{A}_{2}\left(\mathrm{TXA}_{2}\right)$ $\left(\mathrm{TBXA}_{2} \mathrm{R}\right)$ (Supplemental Table 2), while each of these receptors is activated by their cognate ligand in nanomolar ranges $(24,30)$. Also, MaR1 at $10 \mathrm{nM}$ did not appear to activate known SPM receptors for $\mathrm{LXA}_{4}$ (ALX/FPR2), RvE1 (ChemR23/CMKLR1), resolvin D1 (RvD1) (DRV1/G protein-coupled receptor 32 [GPR32]), RvD2 (DRV2/GPR18), and PD1 (GPR37) (31, 32) (Figure 1A and Supplemental Table 2). We confirmed that MaR1 $\left(10^{-13} \mathrm{M}-10^{-7} \mathrm{M}\right)$ did not significantly activate either ALX- $\beta$-arrestin- or GPR32- $\beta$ arrestin-expressing cells, whereas an endogenous ligand of ALX/ FPR2 receptors (ALX) and GPR32, namely RvD1, significantly activated these cells, as a positive control (Supplemental Figure 1C). These results demonstrated receptor specificity for MaR1 compared with other SPM family members.

Because MaR1 exerts potent actions with leukocytes $(10,12)$, we assessed LGR6 expression on human leukocytes using flow cytometry. LGR6 was present on both human peripheral blood PMN and monocytes, but was apparently low on lymphocytes (Supplemental Figure 2A). We also determined LGR6 expression on M1- and M2-like M $\Phi$, because of their pivotal roles in inflammation and its natural resolution (33). LGR6 was present on both M1 and M2 with higher expression on M1-MФ (Supplemental Figure 2B). We assessed whether MaR1 can elicit intracellular $\left[\mathrm{Ca}^{2+}\right]$ release as a second messenger following receptor activation. At $10 \mathrm{nM}$, the functional concentration of MaR1, it did not mobilize intracellular $\left[\mathrm{Ca}^{2+}\right]$ from peripheral blood PMN, monocytes, lymphocytes, or monocyte-differentiated M1- and M2-like MФ (Supplemental Figure 2, C and D).

MaR1 stereoselectivity in activating LGR6. We sought additional $G$ protein-initiated rapid responses following GPCR activation and carried out electrical cell substrate impedance sensing (ECIS). This system monitors rapid changes in impedance initiated by $\mathrm{G}$ protein coupling upon ligand activation of GPCRs (34). With CHO cells overexpressing recombinant human LGR6 (CHO-hLGR6), MaR1 $\left(10^{-11} \mathrm{M}-10^{-7} \mathrm{M}\right)$ dose-dependently reduced impedance, reaching maximum response at about 2 minutes, yielding an $\mathrm{EC}_{50}$ of approximately $0.7 \mathrm{nM}$ (Figure 2, A and B). Using the ECIS system, we tested several structurally related compounds. MaR1-ME was as essentially equipotent as MaR1 in reducing impedance (Supplemental Figure 3, A and B). We also examined protectin D1 (PD1; 10R,17S-dihydroxy-4Z,7Z,11E,13E,15Z,19Z-docosahexaenoic acid) and $\mathrm{LTB}_{4}(5 \mathrm{~S}, 12 \mathrm{R}$-dihydroxy-6Z,8E,10E,14Z-eicosatetraenoic acid) because each contains the dihydroxyl and triene structures with the same configurations (i.e., E,E,Z-conjugated double-bond 
A

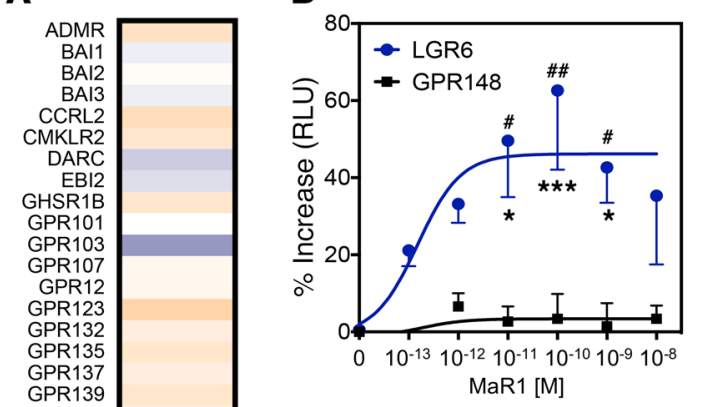

E

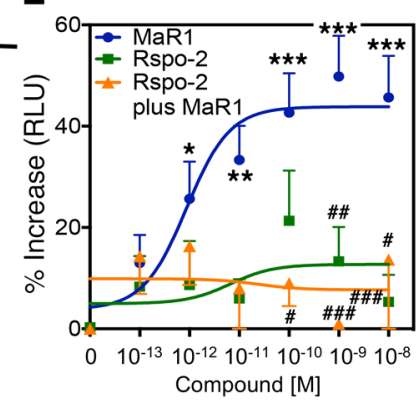

C

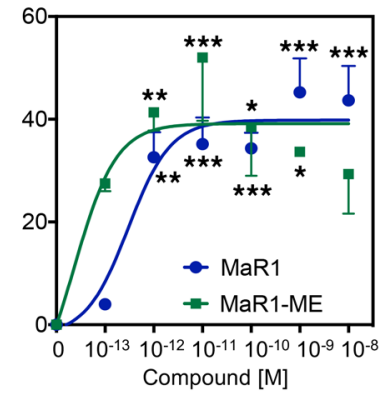

$\mathbf{F}$

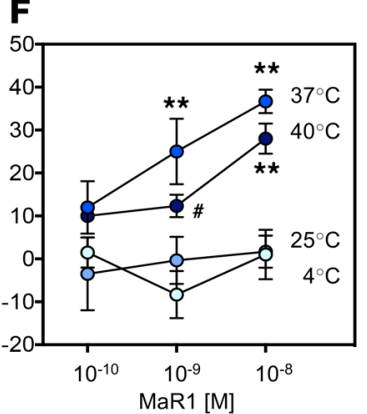

D

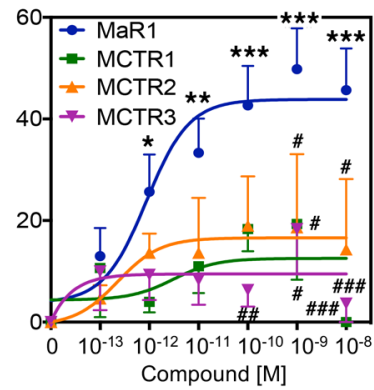

G

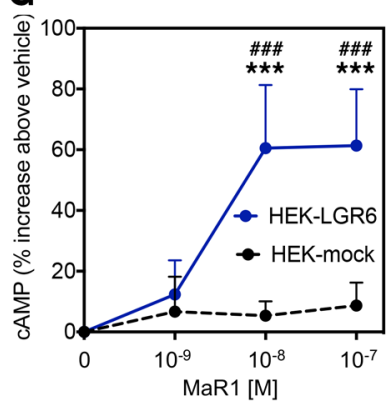

H 14-oxygenation

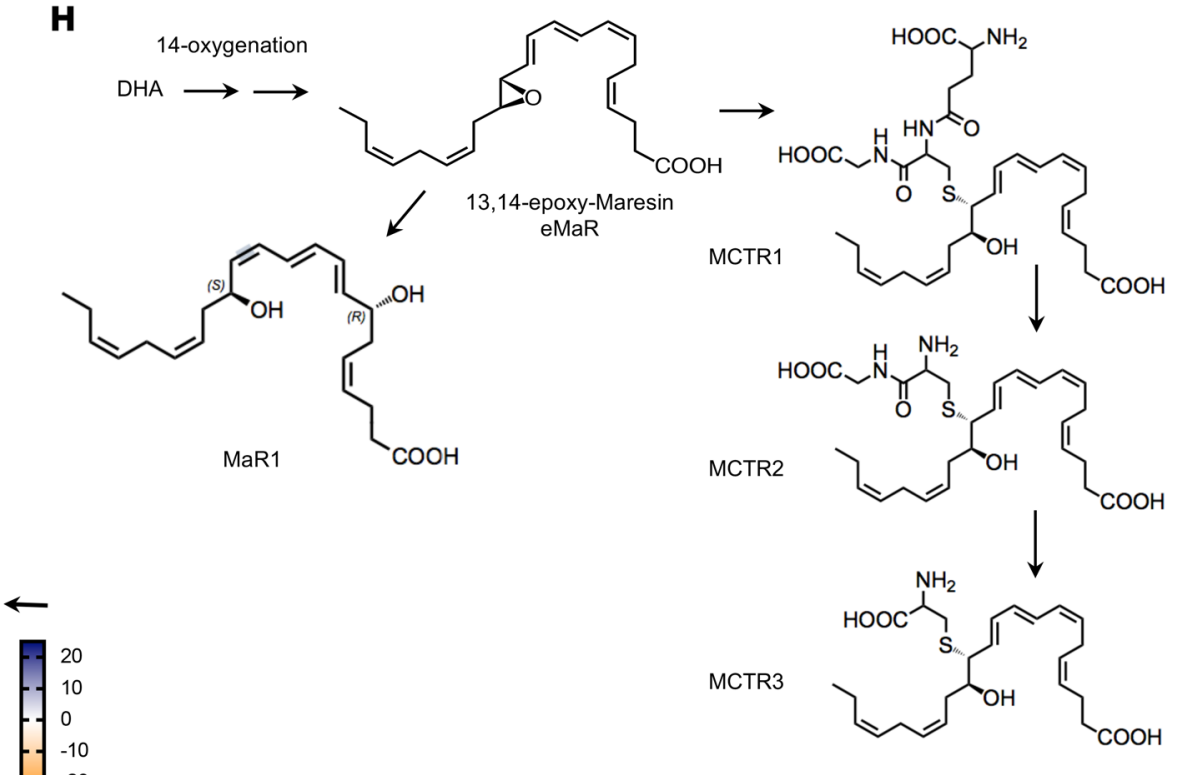

Figure 1. MaR1 candidate receptors. (A) A panel of orphan CPCRs was screened in the presence of $10-\mathrm{nM} \mathrm{MaR1} \mathrm{or} \mathrm{vehicle} \mathrm{(0.1 \%} \mathrm{ethanol)} \mathrm{using} \mathrm{the} \beta$-arrestin PathHunter GPCR system. The $\%$ activity $=100 \% \times$ (mean RLU of test sample - mean RLU of vehicle control)/(mean RLU of vehicle control). (B-E) Ligand (MaR1)-receptor interaction was monitored using the CHO- $\beta$-arrestin system overexpressing LCR6 or CPR148. Results are mean \pm SEM from 3 independent experiments. (B) LCR6 or GPR148 cells with MaR1. ${ }^{*} P<0.05,{ }^{*} P<0.01$. MaR1 versus vehicle with LCR6 cells. ${ }^{*} P<0.05,{ }^{* * *} P<0.001$. LCR6 versus GPR148. (C) LGR6 cells with MaR1 or MaR1 ME. ${ }^{*} P<0.05$, ${ }^{* *} P<0.01 ;{ }^{* *} P<0.001$ versus vehicle. (D) LGR6 cells with MaR1, MCTR1, MCTR2, or MCTR3. ${ }^{*} P<0.05$, ${ }^{*} P<0.01 ;{ }^{* *} P<0.001$ versus vehicle. ${ }^{*} P<0.05$, \#\# $P<0.01 ;{ }^{* \#} P<0.001$ versus MaR1. (E) LGR6 cells with MaR1, Rspo-2, or Rspo-2+MaR1. ${ }^{*} P<0.05$, ${ }^{*} P<$ $0.01 ;{ }^{* * *} P<0.001$ versus vehicle. ${ }^{*} P<0.05,{ }^{\# \#} P<0.01 ; \# \#{ }^{*} P<0.001$ versus MaR1. For $\mathbf{D}$ and $\mathbf{E}$, the 6 groups (MaR1, MCTR1, MCTR2, MCTR3, Rspo-2,

Rspo-2+MaR1) were carried out in the same experiments $(n=3)$. For clarity, the results were separated into $\mathbf{D}$ and $\mathbf{E}$. The same MaR1 response curve is presented in both panels for direct comparisons. The statistical analysis (2-way ANOVA with Tukey's multiple comparisons test) was carried out with all 6 groups. (F) MaR1 (0.1-10 nM) was incubated with CHO- $\beta$-arrestin-LCR6 at $4^{\circ} \mathrm{C}, 25^{\circ} \mathrm{C}, 37^{\circ} \mathrm{C}$, or $40^{\circ} \mathrm{C}$. Results are mean \pm SEM from 3 independent experiments. ${ }^{\#} P<0.05$, versus $4^{\circ} \mathrm{C} ;{ }^{*} P<0.01$, versus $4^{\circ} \mathrm{C}$ and $25^{\circ} \mathrm{C}$. (C) Intracellular cAMP. HEK cells transfected with human LCR6 or mock plasmids were incubated with 1- to $100-\mathrm{nM} \mathrm{MaR1}$ for 15 minutes, and cAMP levels were determined. Results are mean \pm SEM from 4 independent experiments. ${ }^{* * *} P<0.001$, versus HEK-mock cells; ${ }^{\# \# P}<0.001$ versus vehicle control. (B-G) Statistical analysis was carried out using 2-way ANOVA with Tukey's multiple comparisons test.

(H) Maresin biosynthesis pathways. 
A

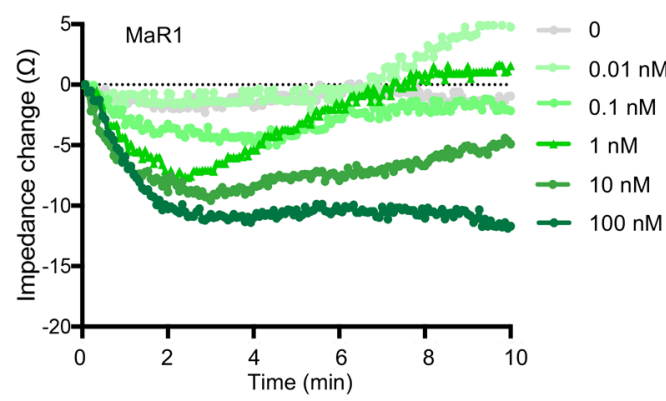

C

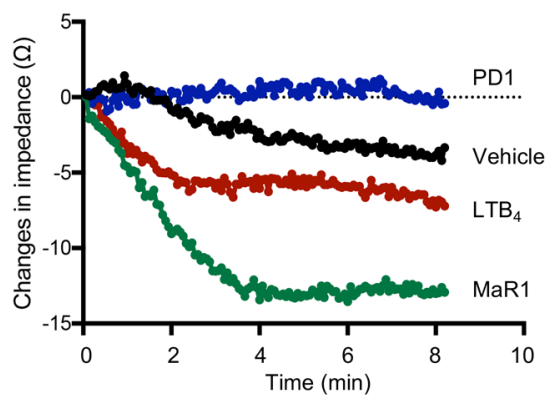

D

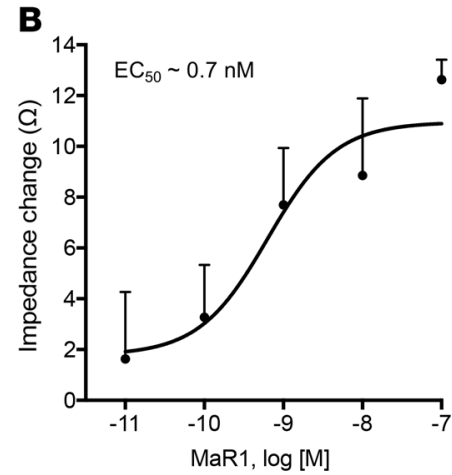

B

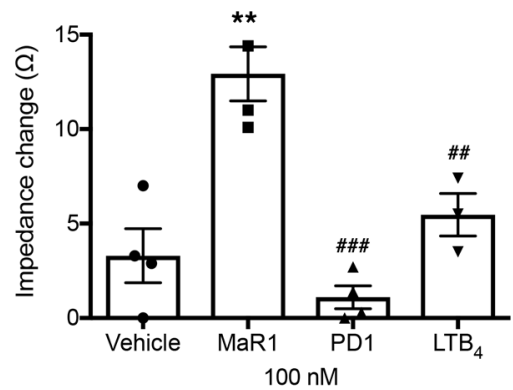

Figure 2. Human recombinant LGR6 receptor specificity. CHO-LGR6 cells were plated onto 8-well ECIS arrays $(8 \mathrm{~W} 10 \mathrm{E}+)$, incubated with (A and B) MaR1 (0.01-100 nM), (C and D) MaR1, PD1, $\mathrm{LTB}_{4}(100 \mathrm{nM})$ or vehicle alone (control), and impedance changes across $\mathrm{CHO}$ cell monolayers were continuously recorded every 4 seconds for 10 minutes using ECIS. Results are mean ( $\mathbf{A}$ and $\mathbf{C}$ ) or mean \pm SEM (B and $\mathbf{D})(n=3-4) .{ }^{* *} P<0.01$, versus vehicle. ${ }^{\# \#} P<0.01 ; \# \# P<0.001$ versus MaR1. One-way ANOVA with Tukey's multiple comparisons test. (E) Space-filling 3D molecular models of MaR1, PD1, and $\mathrm{LTB}_{4}$ with energy minimization. $R$ and $S$ denote the stereochemistry of the hydroxyl groups; $E$ and $Z$ denote the double-bond geometry. Area within the blue dashed lines denotes the dihydroxyl and triene structures oriented in the $E, E, Z$ configuration of these mediators.
E

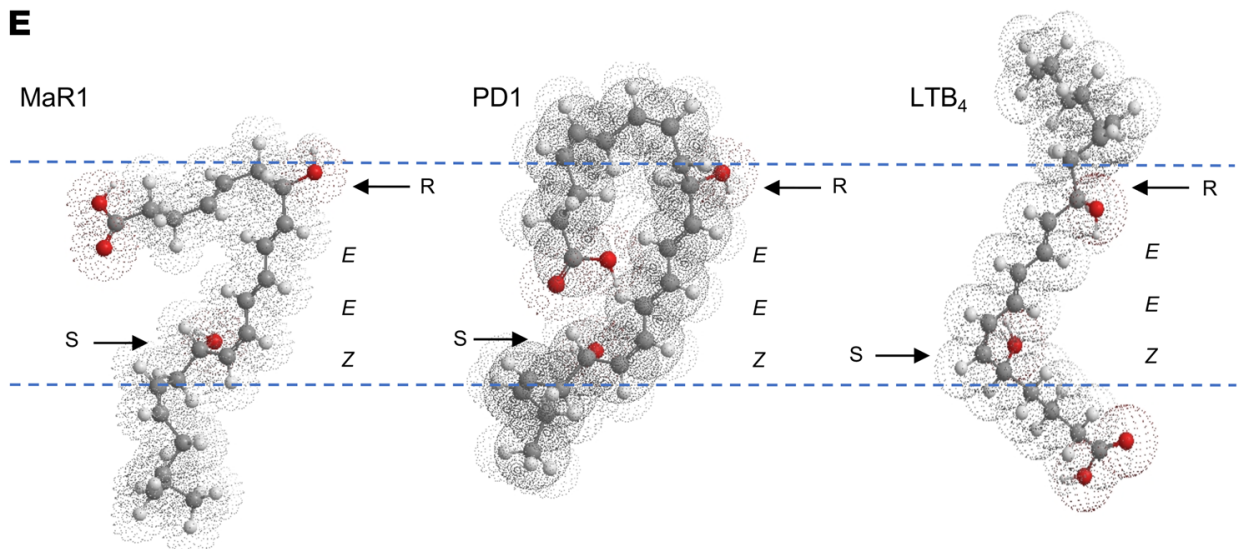

structures) as MaR1 (Figure 2, C-E). The rank order potency was obtained with MaR1>LTB $>>$ PD1 for human recombinant LGR6 (Figure 2D).

Next, we examined the structure-activity relationship with human BLT1, a receptor for the chemoattractant $\mathrm{LTB}_{4}$ (35). With CHO cells overexpressing recombinant human BLT1 (CHO-hBLT1), $\mathrm{LTB}_{4}\left(10^{-12} \mathrm{M}-10^{-7} \mathrm{M}\right)$ dose-dependently increased impedance, giving an apparent $\mathrm{EC}_{50}$ of approximately $0.12 \mathrm{nM}$ (Supplemental Figure 3, C and D), consistent with earlier results for this receptor using ECIS (36). The rank order potency was $\mathrm{LTB}_{4}>>\mathrm{MaR} 1>\mathrm{PD} 1$ for human recombinant BLT1 (Supplemental Figure 3, E and F). These results established the ligand-receptor selectivities for MaR1-LGR6 interactions directly compared with $\mathrm{LTB}_{4}$-BLT1 interactions and demonstrated that these receptor recognitions rely on the overall structures of the mediators, rather than only the di-hydroxy and conjugated triene structures as determinants shared by MaR1, $\mathrm{LTB}_{4}$, and PD1 (Figure 2E).

Because LGR6 is highly expressed in epidermis and dermis, where Lgr6+ cells promote wound repair and digit tip regeneration
$(23,37)$, we addressed whether MaR1 and LGR6 play a role in wound repair with fibroblast and epithelial cells. Human dermal fibroblasts were wounded using electrical field pulse (38), and wound repair from the perimeter into the clearance zone was assessed in real-time by impedance sensing for 6 hours. In this system, MaR1 significantly increased wound repair compared with vehicle-treated cells (Supplemental Figure 4A). A related stereo-isomer, 12E-MaR1 ( $7 R, 14 S$ dihydroxydocosa-4Z,8E,10E,12E,16Z,19Z-hexaenoic acid; structure shown in Supplemental Figure 4A) (12), was less potent than MaR1 at equimolar concentration. To determine if this action of MaR1 was LGR6-dependent, we overexpressed human LGR6 in CHO cells, an epithelial-like cell line derived from the ovary of the Chinese hamster. With these cells (CHO-GFP-LGR6), MaR1 (10-100 $\mathrm{nM}$ ) significantly increased wound repair by $40 \%-80 \%$ above vehicle control in a concentration-dependent fashion (Supplemental Figure 4, B and C). This action of MaR1 was not apparent in GFP mock-transfected CHO cells (Supplemental Figure 4D). Therefore, MaR1 promotes wound repair with epithelial cells in an hLGR6dependent manner. 
A

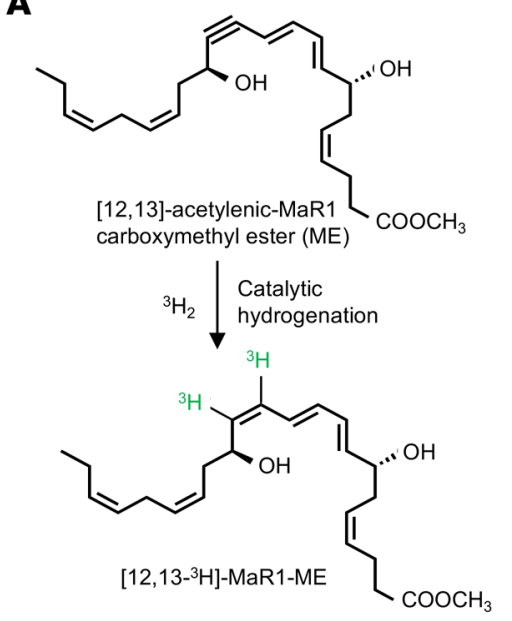

C

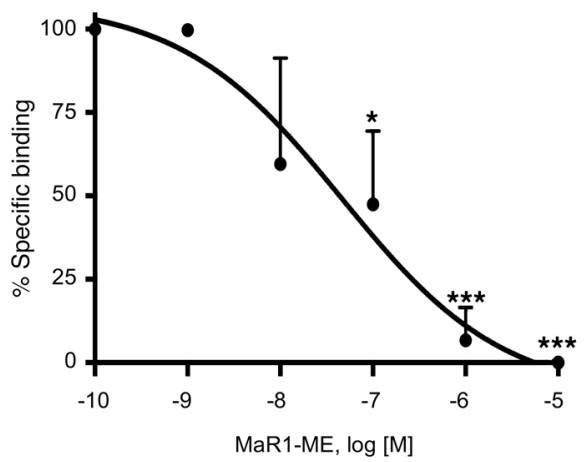

B

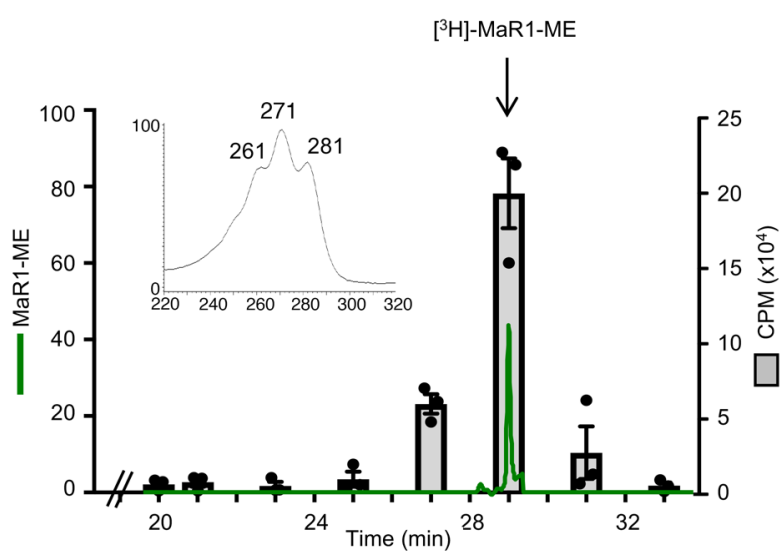

D

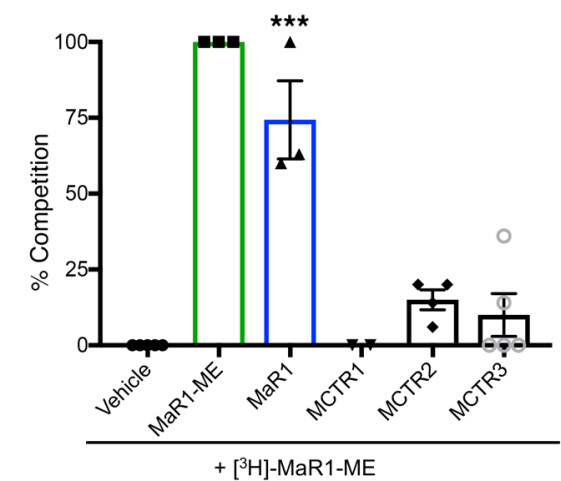

Figure 3. [ $\left.{ }^{3} \mathrm{H}\right]-M a R 1$ preparation and specific binding with human recombinant LGR6. (A) Synthetic [12,13]-acetylenic-MaR1 ME was converted to $\left[12,13-{ }^{3} \mathrm{H}\right]-M a R 1-M E$ via catalytic hydrogenation using tritium gas $\left({ }^{3} \mathrm{H}_{2}\right)$. See Methods. (B) Chromatographic (green line) and radioactive (gray bars) tracing of [ $\left.{ }^{3} \mathrm{H}\right]-\mathrm{MaR} 1-\mathrm{ME}$. Results are mean \pm SEM $(n=3)$. (Inset) Online UV spectra of [ $\left.{ }^{3} \mathrm{H}\right]-\mathrm{MaR} 1-\mathrm{ME} ; \lambda_{\max } 271 \mathrm{~nm}$; representative of 3 separate experiments. (C and D) Competition binding. CHO cells were transfected with a human LGR6 plasmid. Transfected CHO cells $\left(0.5 \times 10^{6}\right.$ cells in $100-\mu \mathrm{I}$ DPBS $\left.{ }^{++}\right)$ were incubated with $2 \mathrm{nM}$ of $\left[{ }^{3} \mathrm{H}\right]-\mathrm{MaR} 1-\mathrm{ME}$ in the absence or presence of (C) increasing concentrations of unlabeled MaR1-ME (10 $\left.0^{-10}-10^{-5} \mathrm{M}\right)$ or $(\mathbf{D})$ unlabeled MaR1-ME (taken as $100 \%$ competition), MaR1, MCTR1, MCTR2 or MCTR3 $\left(10^{-6} \mathrm{M}\right)$ for 60 minutes at $4^{\circ} \mathrm{C}$. Results are mean \pm SEM $(n=3)$. ${ }^{*} P<$ $0.05 ;{ }^{* *} P<0.001$, versus $\left[{ }^{3} \mathrm{H}\right]-\mathrm{MaR} 1-\mathrm{ME}$ plus vehicle. One-way ANOVA with Tukey's multiple comparisons test.

${ }^{3} \mathrm{H}$-MaR1-specific binding. We next determined whether MaR1 can directly bind to recombinant LGR6 using radiolabeled ligand binding. A synthetic precursor 12,13-acetylenic MaR1-ME was prepared, followed by catalytic hydrogenation with tritium gas (see Methods) to generate tritium-labeled $\left[12,13-{ }^{3} \mathrm{H}\right] \mathrm{MaR} 1-\mathrm{ME}$ (Figure $3 \mathrm{~A})$. The integrity of $\left[{ }^{3} \mathrm{H}\right] \mathrm{MaR1}-\mathrm{ME}$ was confirmed and isolated using RP-HPLC. Figure 3B demonstrates the chromatographic tracing of $\left[{ }^{3} \mathrm{H}\right]$ MaR1-ME that coeluted with the authentic synthetic MaR1-ME standard and matched the highest radioactivity peak. In addition, MaR1-ME has a triene structure with characteristic UV bands of absorbance at $\lambda_{\max } 271 \mathrm{~nm}$ with shoulders at 261 and $281 \mathrm{~nm}$, which remained intact in [ $\left.{ }^{3} \mathrm{H}\right] \mathrm{MaR} 1-\mathrm{ME}$ (Figure $3 \mathrm{~B}$, inset). The qualified $\left[{ }^{3} \mathrm{H}\right] \mathrm{MaR1}-\mathrm{ME}$ was then used for specific binding with recombinant human LGR6 expressed in CHO cells. CHO-hLGR6 cells were incubated with [ $\left.{ }^{3} \mathrm{H}\right] \mathrm{MaR} 1-\mathrm{ME}$ in the presence of increasing concentrations of unlabeled MaR1-ME, which dose-dependently competed for $\left[{ }^{3} \mathrm{H}\right]$ MaR1-ME binding (Figure 3C). MaR1 also competed for $\left[{ }^{3} \mathrm{H}\right]$ MaR1-ME binding (Figure 3D). In comparison, MCTR1, MCTR2, or MCTR3 at equimolar concentrations $(1 \mu \mathrm{M})$ do not significantly compete for $\left[{ }^{3} \mathrm{H}\right]$ MaR1ME specific binding (Figure 3D), findings consistent with results obtained with the CHO- $\beta$-arrestin-LGR6 system (Figure 1D). Taken together, these results indicated specific binding of $\left[{ }^{3} \mathrm{H}\right]$ MaR1 with recombinant human LGR6.

Direct action of MaR1 on human PMN in an LGR6-dependent manner. MaR1 exhibits potent actions limiting PMN recruitment to the site of inflammation, a key defining action of proresolving mediators (10). Here, we assessed whether MaR1 has direct actions on PMN chemotaxis using isolated human peripheral blood PMN. We first examined the actions of MaR1 in regulating PMN chemotaxis at the single-cell level in real-time using chemotaxis chambers that provide defined spatiotemporal concentration gradients (see Methods). IL-8 (10 nM) was injected into the microfluidic chambers to form a gradient of the chemoattractant. Isolated human PMN was incubated with vehicle or MaR1 $\left(1\right.$ or $10 \mathrm{nM}$ ) for 10 minutes at $37^{\circ} \mathrm{C}$. PMN chemotaxis toward IL-8 was then recorded for 2 hours in real-time by phase-contrast microscopy and reported in rose plots (Supplemental Figure 5A). MaR1 (1-10 nM) statistically significantly reduced IL-8-initiated chemotaxis by approximately 20\% (Supplemental Figure 5B). Of note, 10-nM MaR1 alone did not significantly stimulate PMN chemotaxis (Supplemental Figure 5C). These results demon- 
A

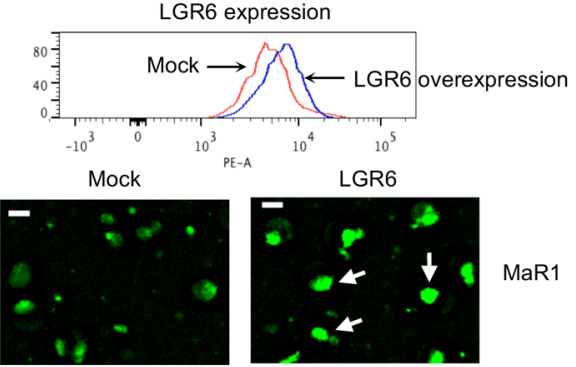

B

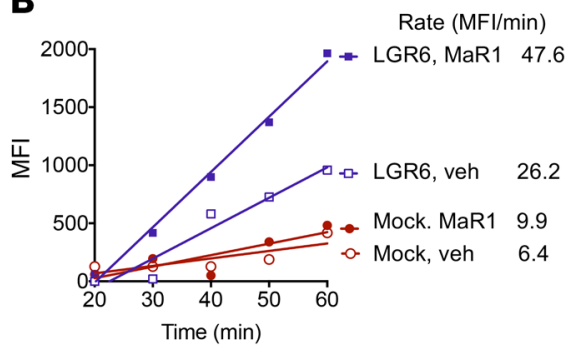

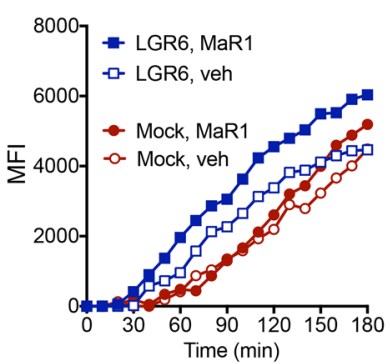

C

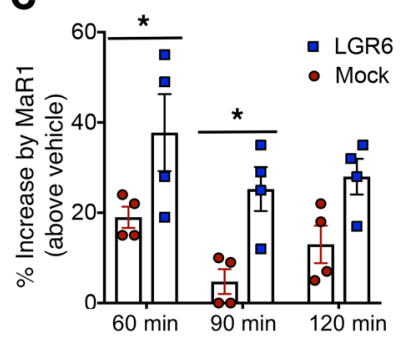

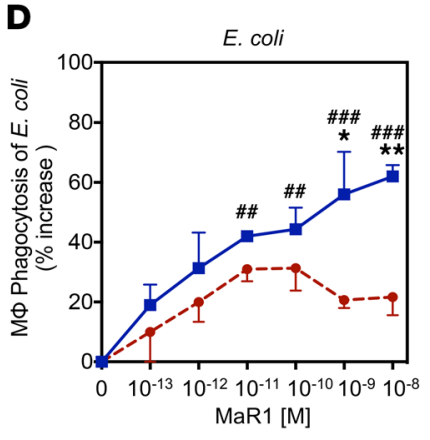

$\mathbf{E}$

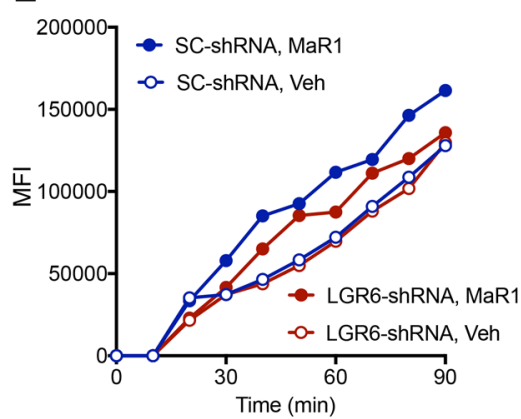

Apoptotic PMN
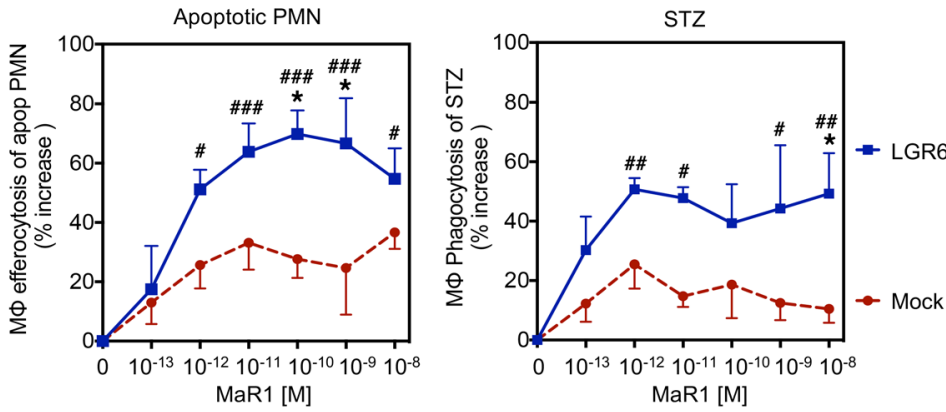

$\mathbf{F}$

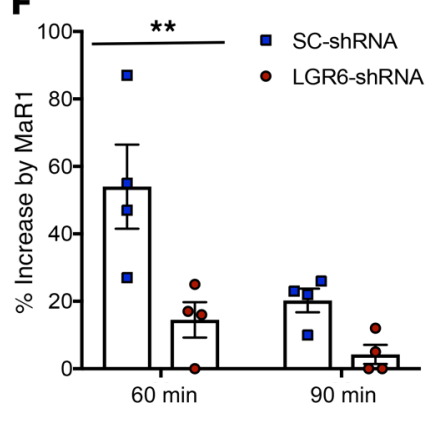

Figure 4. Human LGR6-mediated MaR1 actions on human macrophage phagocytosis: overexpression and knockdown of LGR6. (A-C) Human MФ were transfected with human LGR6 or mock plasmids. Seventy-two hours later, MФ were plated onto chamber slides $\left(0.1 \times 10^{6}\right.$ cells/well), incubated with 1 -nM $\mathrm{MaR} 1$ or vehicle for 15 minutes at $37^{\circ} \mathrm{C}$, followed by addition of BacLight Green-labeled $E$. coli to initiate phagocytosis. Fluorescent images were recorded every 10 minutes. Four separate experiments with separate donors were carried out. In each experiment, 4 fields ( $\times 20)$ per condition (per well) were recorded. (A) (Upper left) LGR6 expression monitored by flow cytometry. (Lower left) Representative fluorescence images. Arrows denote M $\Phi$ with ingested fluorescent $E$. coli. Scale bars: $20 \mu \mathrm{m}$. (Right) mean fluorescence intensity (MFI)/cell from 1 representative experiment. (B) Kinetics of phagocytosis. Rate (MFI/ $\mathrm{min})=\left(\mathrm{MFI}_{60 \mathrm{~min}}-\mathrm{MFI}_{20 \mathrm{~min}}\right) / 40$ min obtained from the same experiment as in (A). (See Supplemental Figure 6 for results obtained from additional 3 donors.) (C) Percent increases of phagocytosis by MaR1. Results are mean \pm SEM $(n=4)$. ${ }^{*}<<0.05$, LGR6 versus mock transfection. (D) Human M $\Phi$ were transfected with human LGR6 or mock plasmids. MФ were incubated with MaR1 $\left(10^{-13}\right.$ to $\left.10^{-8} \mathrm{M}\right)$ or vehicle control for 15 minutes, followed by addition of BacLight Green-labeled E. coli, CFDA-labeled apoptotic PMN, or FITC-labeled STZ. Results are percent increases of phagocytosis above vehicle. Results are mean \pm SEM from 3 independent experiments with separate donors and triplicates in each experiment. ${ }^{*} P<0.05 ;{ }^{* *} P<0.01$, versus mock transfection. ${ }^{\#} P<0.05$, $\# P<0.01 ; \# \# P<0.001$, versus vehicle. (E and F) Human M $\Phi$ were transfected with scramble control (SC)-shRNA or human LGR6-shRNA plasmids and phagocytosis carried out as in $\mathbf{A}$. (E) MFI/cell from 1 representative experiment. (F) Percent increases of phagocytosis by MaR1. Mean \pm SEM $(n=4)$. ${ }^{*} P<$ 0.01, LGR6-shRNA versus SC-shRNA transfections. (C, D, and F) Two-way ANOVA with Bonferroni's multiple comparisons test. 
strate at the single cell level, that MaR1 reduced human PMN migration toward peptide IL-8 gradient chemoattractants.

Next, we examined whether MaR1 regulates PMN chemotaxis in an LGR6-dependent manner. We carried out chemotaxis using the Neuro Probe ChemoTx System in the absence or presence of LGR6 blocking antibodies. In the absence of blocking antibodies, MaR1 (0.1-100 nM) reduced IL-8-initiated PMN chemotaxis approximately 20\%-35\% (Supplemental Figure 5D), comparable with results obtained using real-time chemotaxis chambers (Supplemental Figure 5, A and B). Incubation of PMN with mouse or rabbit anti-human LGR6 antibody before adding MaR1 significantly prevented MaR1-reduced chemotaxis (Supplemental Figure 5D). Together, these results demonstrated direct actions of MaR1 on human PMN, stopping chemotaxis in an LGR6-dependent manner.

LGR6 mediates proresolving actions of MaR1 on human MФ. Because MaR1 enhances M $\Phi$ phagocytosis (10), a cellular hallmark of tissue resolution of acute inflammation (1), we examined whether this action was LGR6 dependent. Human MФ were differentiated from peripheral blood monocytes (see Methods), transfected with either a mock or human LGR6 plasmid, and LGR6 expression was verified using flow cytometry (Figure 4A). Phagocytosis of fluorescent-labeled E. coli was monitored in real-time using fluorescent microscopy. MaR1 $(1 \mathrm{nM})$ increased E. coli uptake with mock-transfected MФ. This action was further enhanced with hLGR6 transfection (Figure 4A, representative images and real-time recordings). We also monitored the rate of phagocytosis from 20-60 minutes. In a representative set of experiments, mock-transfected MФ ingested $E$. coli with a rate of approximately 6.4 MFI/min, and 1-nM MaR1 increased the rate to approximately 9.9 MFI/min. This action was enhanced with hLGR6 transfection, with a rate of approximately $26.2 \mathrm{MFI} / \mathrm{min}$ with $E$. coli alone and approximately 47.6 MFI/min with E. coli + MaR1 (Figure 4B). The phagocytosis time course (0-120 min) and initial kinetics (20-60 min) for an additional 3 donors are shown in Supplemental Figure 6. Also, MaR1 increased phagocytosis approximately $40 \%$ at 60 minutes and approximately $30 \%$ at 90 to 120 minutes with LGR6-transfected cells, which were significantly higher than the increases with mock-transfected cells (Figure 4C; $n=4$ ).

It is noted that LGR6-transfected M $\Phi$ gave higher phagocytic activity than mock-transfected cells in the absence of exogenous MaR1. Thus, we questioned whether there was endogenous production of MaR1 that affected phagocytosis in transfected human MФ (Supplemental Figure 7 and Supplemental Table 3). We determined LM/SPM levels using mass spectrometry-based metabolipidomics (See Methods). In both mock- and LGR6-transfected $\mathrm{M} \Phi$, select SPM were present, including MaR1, RvD2, and RvD5. There were no significant differences of MaR1 and total SPM levels between mock- and LGR6-transfected MФ in the absence or presence of $E$. coli. The endogenous MaR1 levels $\left(\sim 5 \mathrm{pg} / 10^{6}\right.$ cells/ ml; 10 pM; Supplemental Figure 7) are within its bioactive concentration ranges $(7,15-19)$. Thus, it is possible that endogenous MaR1 interactions with overexpressed LGR6 in LGR-transfected M $\Phi$ gave heightened phagocytosis compared with mock-transfected MФ (Figure 4, A and B and Supplemental Figures 6 and 7).

To further investigate the role of LGR6 in MaR1-stimulated phagocytosis and efferocytosis, MФ ingestion of fluorescent- labeled E. coli, apoptotic PMN, or serum-treated zymosan (STZ) were carried out in mock- or LGR6-transfected MФ, and fluorescence was monitored using a fluorescence plate reader. MaR1 (10 pM-10 nM) significantly increased MФ phagocytosis of BacLight green-labeled E. coli or CFDA-labeled apoptotic PMN in LGR6-transfected MФ, compared with mock transfections (Figure $4 \mathrm{D} ; \sim 60 \%$ increase above vehicle in M $\Phi$-hLGR6 versus $~ 20 \%$ increase in MФ-mock; 1-nM MaR1). LGR6 overexpression also gave heightened phagocytosis of FITC-labeled STZ in response to MaR1 (1 pM-10 nM), compared with mock transfections (Figure 4D). Thus, LGR6 overexpression in human MФ enhanced MaR1's action in stimulating phagocytosis of E. coli, apoptotic PMN, and STZ. In separate sets of experiments, knockdown of endogenous MФ LGR6 using specific shRNA (LGR6-shRNA) significantly reduced MaR1-stimulated phagocytosis of E. coli, compared with M $\Phi$ transfected with scramble control shRNA (SC-shRNA) (Figure 4, $\mathrm{E}$ and $\mathrm{F}$ ). Together, these results demonstrated that human LGR6 contributed to proresolving actions of MaR1 by stimulating $\mathrm{M} \Phi$ ingestion of yeast zymosan particles, live E. coli, as well as apoptotic human PMN.

Because MaR1 partially interacted with recombinant human BLT1 (Supplemental Figure 3, E and F), we examined whether a BLT1 antagonist can have an impact on MaR1-simulated MФ phagocytosis. BLT1 antagonist U75302 alone (100 nM) did not significantly alter phagocytosis, nor did it affect 10-nM MaR1-stimulated phagocytosis in nontransfected, SC-shRNA and LGR6shRNA-transfected MФ (Supplemental Figure 8). These results suggest that MaR1's action in stimulating $\mathrm{M} \Phi$ phagocytosis is not dependent on the human BLT1 receptor. Because LGR6, Rspo-2 reduced MaR1-initiated activation of CHO- $\beta$-arrestin-LGR6 cells (Figure 1E), we examined whether Rspo-2 altered MaR1's proresolving functions with МФ. At equal molar concentrations, 10-nM Rspo-2 did not significantly enhance phagocytosis but significantly reduced MaR1-stimulated phagocytosis with human MФ (Supplemental Figure 9). These results support those obtained with recombinant LGR6 (Figure 1E) and suggest that Rspo-2 blocks MaR1 signals and functions.

Knockdown of LGR6 diminishes MaR1 binding and functions on human THP-1 cells. To further investigate LGR6-dependent MaR1 signals and pro-resolving functions, we established a stable LGR6-deficeint THP-1 cell line (human monocyte-like cells) using a lentivirus-based system (Figure 5A). In order to determine the LGR6 shRNA knockdown efficiency, we transfected HEK cells with 3 different types of hairpin shRNA (See Supplemental Table 4). HEK-shRNA LGR6.1 cells had a greater than $65 \%$ gene knockdown compared with a mock vector (denoted SGEP; ref. 49), Ren.713 control, shRNA LGR6.2, or LGR6.3 (Supplemental Figure 10, A-C). Therefore, the shRNA LGR6.1 construct was selected to transfect THP- 1 cells. The transfected THP-1 cells were sorted to select $\mathrm{GFP}^{+}$populations for further investigation. In LGR6 shRNA-transfected cells $\left(\mathrm{GFP}^{+}\right.$, see gating strategy in Figure 5A), LGR6 expression was reduced more than 50\%, compared with THP-1 transfected with a mock vector or a control shRNA Ren.713 (Figure 5B). These cells were examined for their specific binding with $\left[{ }^{3} \mathrm{H}\right]$ MaR1-ME. In THP-1 cells transfected with a mock vector, MaR1 competed for $\left[{ }^{3} \mathrm{H}\right]$ MaR1-ME binding, giving statistically significant specific binding. In cells transfected with 
A LGR6 knockdown

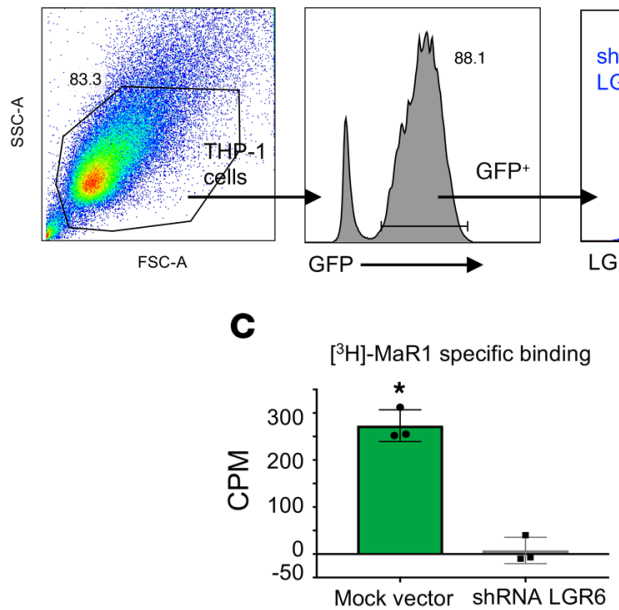

B

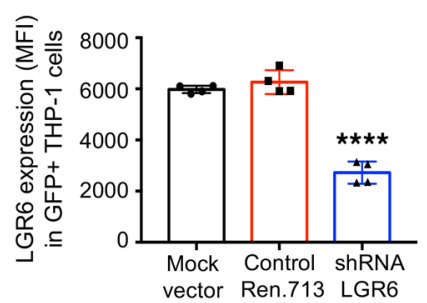

E

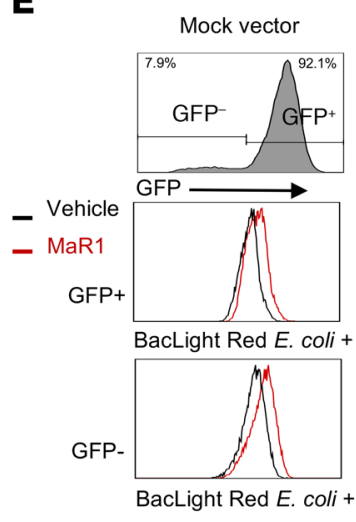

G

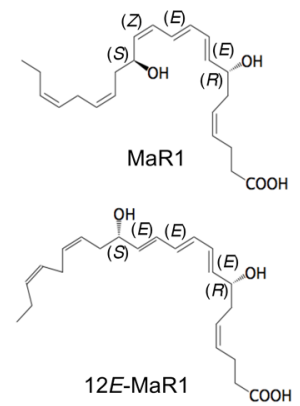

Control Ren.713
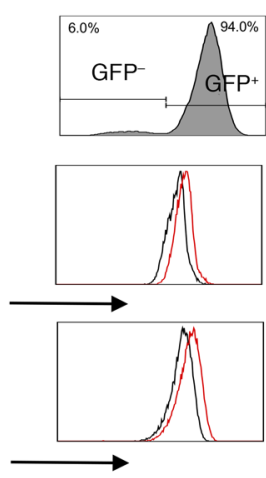

Mock vector

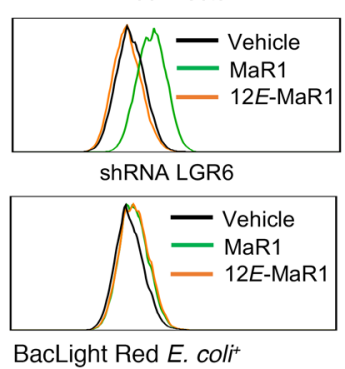

shRNA LGR6
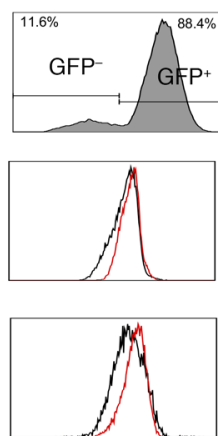

H
$\mathbf{F}$

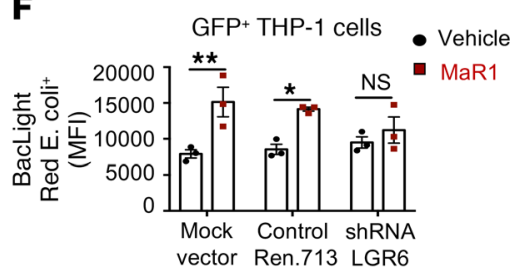

GFP- THP-1 cells

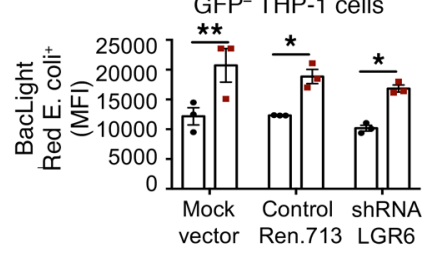

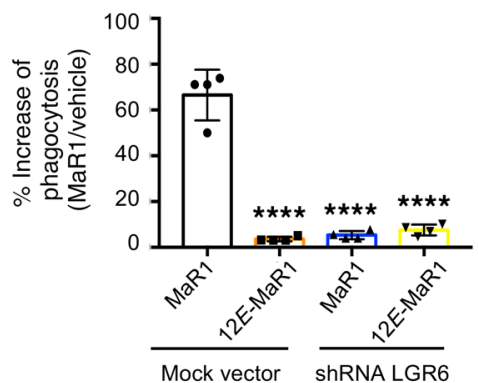

Figure 5. MaR1-LGR6-dependent binding, signal, and phagocytosis: knockdown of human LGR6. (A and B) LGR6 knockdown in THP-1 cells. (A) Gating strategy: Cells were gated on FSC-SSC dot plots (left), GFP+ populations were selected on the histograms (middle), then LGR6 expression was determined within the GFP+ ${ }^{+}$population (right). (B) LGR6 expression (MFI) in GFP+ THP-1 cells. Results are mean $\pm \mathrm{SEM}(n=4)$. ${ }^{* * *} P<0.001$ versus control shRNA (Ren 713) and mock vector. (C) [ $\left.{ }^{3} \mathrm{H}\right]-\mathrm{MaR} 1$ binding. THP-1 cells were incubated with 2-nM [ $\left.{ }^{3} \mathrm{H}\right]-\mathrm{MaR} 1-\mathrm{ME}$ in the presence or absence of unlabeled 1- $\mu \mathrm{M}$ MaR1ME for 60 minutes at $4^{\circ} \mathrm{C}$. Results are specific binding (CPM), calculated as total CPM ([ $\left.{ }^{3} \mathrm{H}\right]-\mathrm{MaR} 1$ plus vehicle) - nonspecific CPM ([ $\left.{ }^{3} \mathrm{H}\right]-\mathrm{MaR} 1 \mathrm{plus}$ unlabeled MaR1-ME). Results are mean \pm SEM $(n=3) .{ }^{*} P<0.05$, versus shRNA LGR6. Two-tailed paired Student's $t$ test. (D) CAMP. THP-1 cells were incubated with MaR1 (1-100 nM) for 15 minutes and cAMP levels determined. Results are mean $\pm \operatorname{SEM}(n=3) .{ }^{*} P<0.01$, versus shRNA LGR6; \#\# $P<0.01$, versus 1 $\mathrm{nM}$ (mock vector). (E-H) Phagocytosis. THP-1 cells were incubated with 10-nM MaR1 or vehicle (0.01\% ethanol) for 15 minutes prior to addition of BacLight Red-labeled (PE-Texas Red) E. coli (1:50 THP-1:E.coli) for 45 minutes at $37^{\circ} \mathrm{C}$. Flow cytometry was carried out. (E) Representative histograms for GFP (top panels), GFP+ BacLight Red E. coli+ (middle panels) and GFP- BacLight Red E. coli+ (bottom panels). (F) Quantification of BacLight Red E. coli (MFI) in GFP+ cells (top) and GFP- cells (bottom). Results are mean $\pm \operatorname{SEM}(n=3) .{ }^{*} P<0.05$; ${ }^{* *} P<0.01$, MaR1 versus vehicle. (G) Structures of MaR1 and 12E-MaR1 (left) and representative histograms (right) of BacLight Red E. coli in GFP+ cells. (H) Percent increase of phagocytosis. ${ }^{* * *} P<0.0001$, versus MaR1-treated mock vector transfected cells. (B and $\mathbf{H}$ ) One-way ANOVA or ( $\mathbf{D}$ and $\mathbf{F}$ ) 2-way ANOVA with Tukey's multiple comparisons test. 
shRNA-LGR6, specific binding to $\left[{ }^{3} \mathrm{H}\right]$ MaR1-ME was diminished (Figure 5C). In addition, 10- to 100-nM MaR1 significantly increased cAMP levels by approximately $60 \%$ (Figure 5D), consistent with results obtained with HEK cells overexpressing LGR6, where 10- to 100-nM MaR1 also increased cAMP about $60 \%$ (Figure 1G). This stimulation of cAMP by MaR1 was diminished in THP-1 transfected with shRNA targeting LGR6 (Figure 5D).

We next carried out phagocytosis of E. coli with transfected THP-1 cells. MaR1 (10 nM, 15 min) was incubated with THP-1 cells, followed by addition of BacLight Red-labeled E. coli for 45 minutes. BacLight Red-labeled E. coli fluorescence intensities were then monitored using flow cytometry, representing $\mathrm{M} \Phi$ phagocytosis of E. coli. In the transfected cells (GFP based shRNA expression vector miRE-SGEP), approximately $90 \%$ of the total cells were GPF (see gating strategy in Figure 5, A and E). MaR1 increased more than $40 \%$ BacLight Red E. coli intensities in $\mathrm{GFP}^{+}$ populations in both mock and control Renila 713 shRNA-transfected cells (Figure 5, E and F). In contrast, in shRNA-LGR6-transfected cells, MaR1 did not significantly enhance BacLight Red $E$. coli intensities in $\mathrm{GFP}^{+}$cells (Figure 5E). In comparison, in GFPpopulations, MaR1 retained its function in increasing phagocytosis (i.e., BacLight Red E. coli intensities) in shRNA-LGR6 as well as in control plasmid-transfected cells (Figure 5E). These results clearly demonstrated LGR6-dependent MaR1 functions in stimulating phagocytosis of $E$. coli with THP-1 cells.

To address stereoselectivity of MaR1-LGR6 interactions, we carried out phagocytosis with MaR1 in comparison with a stereoisomer 12E-MaR1 (7R,14S-dihydroxydocosa-4Z,8E,10E,12E,16Z, 19Z-hexaenoic acid; structure shown in Figure 5G). 12E-MaR1 at 10 $\mathrm{nM}$ did not significantly increase phagocytosis, indicating that the conjugated triene of MaR1 in $8 E, 10 E, 12 Z$ configuration is essential for activating LGR6 (Figure 5, G and H). Together, results obtained from LGR6 overexpression and knockdown indicated that MaR1 elicited stereoselective and LGR6-dependent pro-resolving functions with human phagocytes.

MaR1-LGR6 initiated signals on phagocytes. Next, we investigated MaR1-initiated intracellular signals with human MФ on the single-cell level using time-of-flight mass cytometry (СуTOF). M1- and M2-MФ were each incubated with 10-nM MaR1 for 0-15 minutes and levels of a panel of phospho-proteins determined (see Methods). MaR1 at $10 \mathrm{nM}$ time-dependently increased phosphorylation of pp38MAPK and pCREB with both M1- and M2-MФ with different kinetics (Figure 6A). For example, MaR1 rapidly and transiently increased pp38MAPK and pCREB phosphorylation within 1 minute with M2-M proteins reached maximum at 2 minutes with M1-MФ. CyTOF was also carried out with peripheral blood leukocytes (compare with ref. 39). MaR1 increased phosphorylation of pERK and pCREB, giving the strongest signals in PMN and $\mathrm{CD} 14^{+}$classical monocytes followed by $\mathrm{CD}_{16}{ }^{+}$non-classical monocytes and dendritic cells. These results indicate on the single-cell level that MaR1 elicited rapid and transient protein phosphorylation as intracellular signals in peripheral blood leukocytes and isolated MФ.

Next, we investigated whether these MaR1-initiated intracellular signals are LGR6-dependent. MaR1 (10 nM) was incubated with THP-1 cells transfected with a mock vector or LGR6-specific shRNA for 0-5 minutes (Figure 6, B and C). In mock-transfected cells, MaR1 time-dependently increased phosphorylation of pERK and PCREB, reaching maximum at 1 and 2 minutes, respectively. This action was abolished in shRNA-LGR6-transfected cells (Figure 6, B and C). In addition, 12E-MaR1, the trans double-bond isomer of MaR1, did not statistically significantly increase phosphorylation of pCREB and pERK (Figure 6, D and E), consistent with results obtained with THP-1 phagocytosis (Figure 5, G and H) and fibroblast wound repair (Supplemental Figure 4A). Taken together, these results indicate that MaR1 regulated phosphorylation of select kinases and transcription factors with different kinetics in THP1 cells in an LGR6-dependent manner.

MaR1-LGR6 interaction in vivo reduces exudate PMN. We examined whether knockdown of endogenous mouse LGR6 can enhance MaR1's action in vivo using a self-limited murine peritonitis. We injected LGR6-specific siRNA or nontarget siRNA $(10 \mu \mathrm{g} /$ mouse) i.p. Three days later, peritonitis was initiated with zymosan. MaR1 was given (100 ng/mouse, i.p.) at peak of inflammation (12 hours after zymosan administration), and exudates were collected at 24 hours. Intraperitoneal administration of LGR6 siRNA significantly decreased LGR6 expression, yielding an approximately $23 \%, 30 \%$, and $33 \%$ reduction in exudate PMN, monocytes, and $\mathrm{M} \Phi$, respectively (Figure 7, A and B). Administration of MaR1 significantly reduced PMN numbers at 24 hours $(\sim 70 \%)$ in mice given nontarget siRNA. In comparison, MaR1's actions were significantly reduced in mice that received LGR6 siRNA, giving an approximately $26 \%$ decrease in exudate PMN (Figure 7C). These results indicate that MaR1-LGR6 interactions limited PMN and promoted resolution of inflammation.

To address direct MaR1-LGR6 actions on specific leukocytes, we injected LGR6-specific siRNA or nontarget siRNA $(10 \mu \mathrm{g} /$ mouse) i.p. and collected peritoneal MФ 3 days later to assess their phagocytic activity. M $\Phi$ from LGR6-siRNA-injected mice reduced LGR6 expression by more than 50\% compared with nontarget siRNA-injected mice (Figure 7D). LGR6 knockdown in MФ significantly reduced MaR1-stimulated phagocytosis of E. coli as shown in Figure 7, E and F. These results indicate that in vivo silencing of LGR6 reduced MaR1-stimulated phagocytosis functions with mouse peritoneal MФ.

We also collected peripheral blood from these mice to assess MaR1-LGR6-dependent signals on PMN and monocytes. LGR6-siRNA injection reduced LGR6 expression in PMN and monocytes by $49 \%$ and $46 \%$, respectively, compared with nontarget siRNA-injected mice (Figure 8A). MaR1 at $10 \mathrm{nM}$ initiated time-dependently increased phosphorylation of ERK and CREB in PMN ( 25\%-30\% increase at 1 minute; Figure 8, B-D) and monocytes ( $20 \%-30 \%$ increase at 1 minute; Figure $8, E-G)$ collected from mice that received nontarget siRNA. These actions were statistically significantly reduced in PMN, and monocytes collected from mice received LGR6-siRNA. Thus, LGR6 silencing in vivo reduced MaR1-initiated phosphorylation signals in mouse peripheral blood phagocytes.

\section{Discussion}

Herein, we carried out unbiased screening with MaR1 of human receptors and identified LGR6. We established activity of MaR1 with recombinant human LGR6 receptor using GPCR- $\beta$-arrestinbased receptor activation and ligand-GPCR-initiated imped- 
A
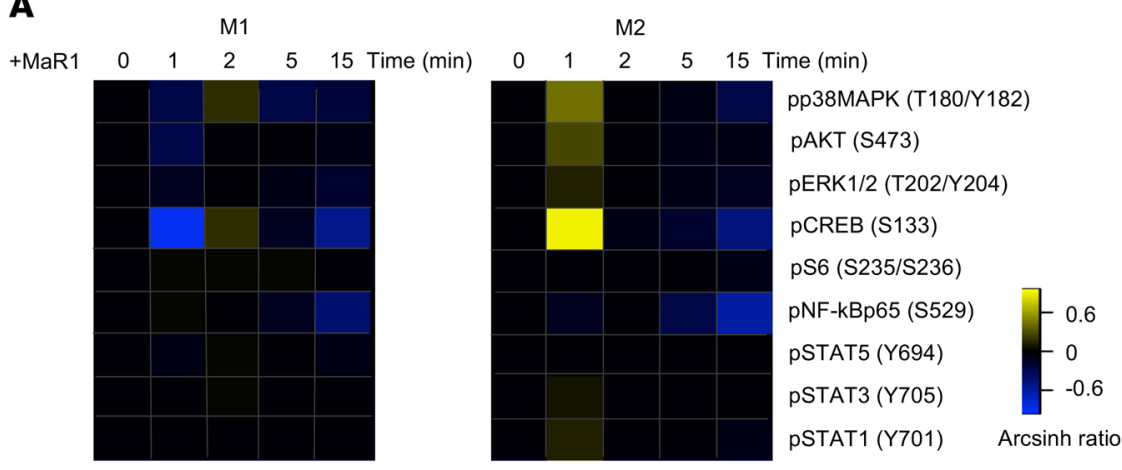

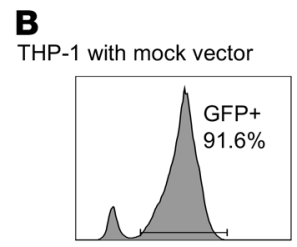

THP-1 with shRNA LGR6

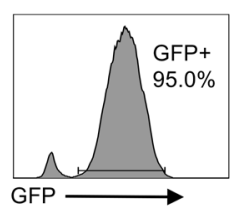

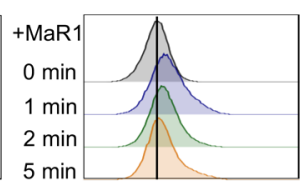

pERK $1 / 2$

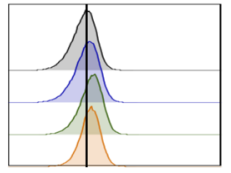

pCREB

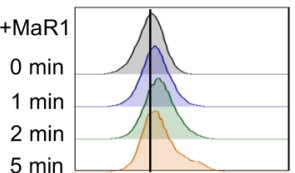

pERK 1/2

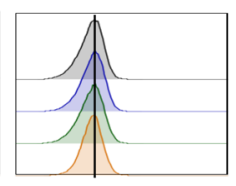

pCREB
C

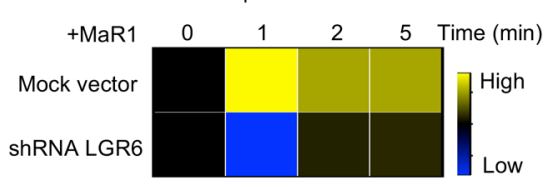

pCREB

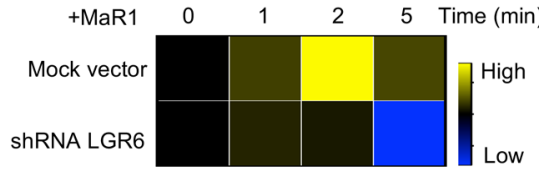

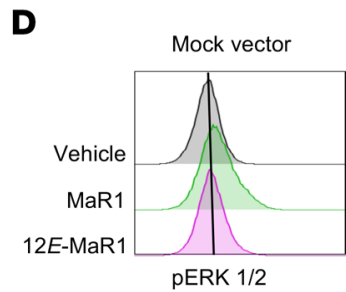

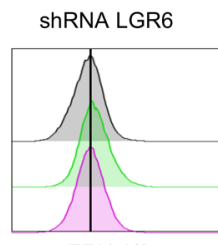

Mock vector

shRNA LGR6

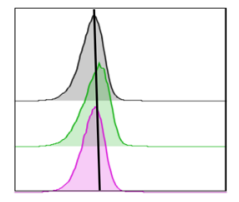

pCREB

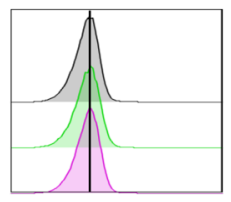

pCREB
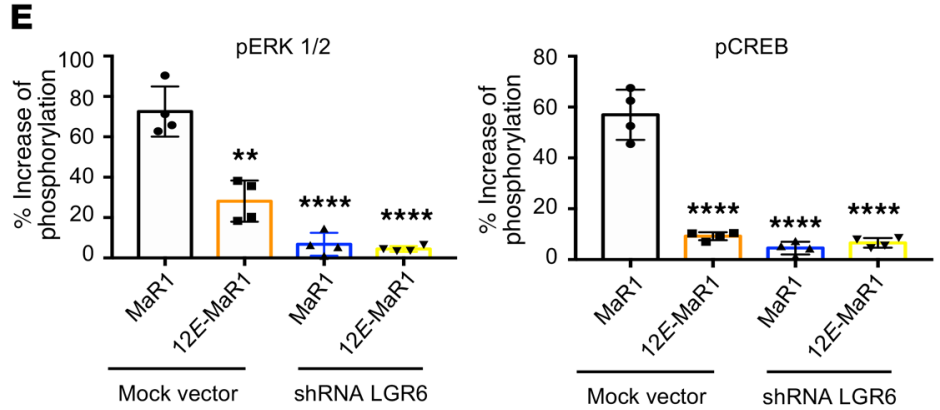

Figure 6. MaR1-LGR6-dependent phosphorylation signals. (A) MaR1-dependent protein phosphorylation in human macrophages. Heat maps of phosphorylated signaling molecules at 0, 1, 2, 5, and 15 minutes after exposure of 10-nM MaR1 in M1 and M2 human macrophages was obtained using CyTOF (see Methods). (B, C) THP-1 cells transfected with either a mock vector or LGR6-specific shRNA were incubated with 10 -nM MaR1 for 0 to 5 minutes. pCREB and pERK levels in GFP cells were determined using flow cytometry. Results are (B) representative histograms and (C) heat maps from $n=4$. ( $\mathbf{D}$ and E) Comparisons of MaR1 and its 12E isomer. THP-1 cells transfected with either a mock vector or LGR6-specific shRNA were incubated with MaR1 or 10-nM 12E-MaR1 for 1 (for pERK) or 2 minutes (for pCREB). pCREB and pERK levels were determined using flow cytometry. Results are (D) representative histograms and (E) mean \pm SEM from 4 independent experiments. ${ }^{* *} P<0.01$; ${ }^{* * *} P<0.0001$ versus MaR1-treated mock vector transfected cells. One-way ANOVA with Tukey's multiple comparisons test.

ance changes. To obtain direct evidence for specific binding with recombinant LGR6, we prepared synthetic radiolabeled MaR1 that gave stereoselective and noncovalent recognition of this receptor. Given these results, we obtained evidence for LGR6-mediated proresolving functions of MaR1, namely, stimulating МФ phago- cytosis and phagocyte signaling via overexpression or knockdown of human and mouse LGR6.

LGR6 is a member of the glycoprotein hormone receptor subfamily of rhodopsin-like GPCR. The transcripts of LGR6 are highly expressed in the testis, ovary, and small intestine, 
followed by the oviduct, uterus, colon, spleen, kidney, heart, and brain (40), organs known to contain macrophages, monocytes, and PMN. Using flow cytometry, we found that LGR6 was present on the cell surface of immune cells including human M1- and M2-M $\Phi$, peripheral blood PMN, and monocytes (Supplemental Figure 2A). Consistent with this expression pattern of LGR6 on phagocytes, MaR1 enhanced phagocytosis with $\mathrm{M} \Phi$ (Figure 4 and Figure 7) and prominent phosphorylation signals with PMN and monocytes (Figures 6 and 8), as well as reduced chemotaxis with human PMN (Supplemental Figure 5) in LGR6-dependent manners.

In the maresin family, other members that stimulate regeneration and are proresolving (namely MCTR1, MCTR2, and MCTR3) did not directly activate LGR6 (Figures 1 and 3). Earlier, we demonstrated that MCTRs interact with human cysteinyl-LT receptor CysLT1 to reduce LTD $_{4}$ signals (41). CysLT1 is highly expressed on endothelial and smooth muscle cells (24). Consistent with this

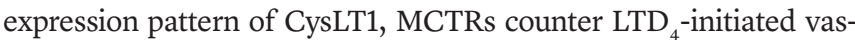
cular and smooth muscle responses (41). In comparison, MaR1 activated LGR6, initiating rapid downstream signals, that is, cAMP and impedance change (Figure 1 and Figure 2), as well as stimulating innate immune responses on PMN, monocytes, and $\mathrm{M} \Phi$ (Figures $4-8$ ). These results suggest the family and pathway specificity for MaR1 versus cysteinyl-containing MCTRs (see biosynthetic scheme in Figure $1 \mathrm{H}$ ) in initiating distinct signals via separate and specific receptors, regulating different pathways in resolution of inflammation with specific cell types and microenvironments.

LGR6 is related to LGR4 and LGR5, sharing approximately $45 \%$ identity in deduced amino acid sequences (40). LGR6 and LGR5 mark adult stem cells in multiple tissues. LGR4 and LGR5 were not activated by MaR1 within the screened panel of orphan GPCRs (Figure 1A). LGR6 is highly expressed in epidermal stem cells, contributing to long-term skin regeneration and repair, including formation of new hair follicles (23), generating taste bud cells (42), and digit tips (37). R-spondins can bind LGR4, LGR5, and LGR6 to potentiate Wnt/ $\beta$-catenin signaling (14). However, LGR6 is apparently not coupled to heterotrimeric G proteins or $\beta$-arrestins following R-spondin binding (38). Along these lines, it was recently reported that R-spondins exhibit LGR-independent Wnt responsiveness and instead function as direct antagonists of RNF43 and ZNRF3 proteins that govern limb development (43). Herein, we found that Rspo-2, in the same concentration ranges as MaR1, did not activate CHO- $\beta$-arrestin-LGR6 cells, but rather blocked MaR1 actions on these cells (Figure 1E). Also, Rspo-2 significantly reduced MaR1-stimulated phagocytosis (Supplemental Figure 9). These results suggest that MaR1 and Rspo-2 may share some recognition sites on LGR6, yet evoke different signals and biological functions.

Selectivity of MaR1 with human LGR6 was evaluated using ligand-GPCR-initiated $\beta$-arrestin recruitment and functional sensing (i.e., ECIS) as well as specific radiolabel binding (Figures 1-3). For structural specificity, both MaR1 and MaR1-ME directly bind and activate LGR6 (Figure 1C, Figure 3D, and Supplemental Figure 3, A and B). Of interest, the MEs of LXA, RvD1, and RvD2 also bind and activate their cognate receptors ALX, GPR32, or DRV2/ GPR18 (31). This finding contrasts with prostaglandin receptors, for which prostaglandin carboxylic acids are essential for receptor activation $(24,30)$. A stereoisomer $12 E-M a R 1$, formed naturally by nonenzymatic hydrolysis of the epoxide intermediate eMaR, did not stimulate either wound repair or macrophage functions (Figure 5, Figure 6, and Supplemental Figure 4A). On the receptor side of the interactions, MaR1 did not significantly activate the other currently known SPM receptors, such as ALX/FPR2, ChemR23/ CMKLR1, DRV1/GPR32, DRV2/GPR18, and GPR37 (Figure 1A, Supplemental Figure 1, and Supplemental Table 2). These specific SPM-receptor interactions initiate overlapping and distinct signals as well as proresolving functions, acting in tandem to govern host immune response. It is possible that compensatory mechanisms may exist among these SPM-receptor axes to ensure proper host defense against injury and infection and its timely resolution.

LGR6-dependent MaR1 signals and functions were documented in recombinant systems and with human phagocytes. MaR1 increased cAMP, a second messenger downstream of $G \alpha$, in LGR6-expressing HEK cells and in the monocytic cell line THP-1 cells, that was diminished by LGR6 knockdown (Figure $1 G$ and Figure 5D), suggesting that LGR6 is likely to couple to $G \alpha_{\text {s }}$ protein upon activation by MaR1. These results are consistent with those obtained using impedance sensing (Figure 2). This cell-based and radiolabel-free system is able to quantitatively determine direct GPCR activation and distinguish $G \alpha_{s}, G \alpha_{i}$, and $\mathrm{G} \alpha_{\mathrm{q}}$ coupling. $\mathrm{G} \alpha_{\mathrm{i}}$-coupling and $\mathrm{G} \alpha_{\mathrm{s}}$-coupling give rapid increase and decrease of impedance, respectively (34). The $\mathrm{LTB}_{4}$ receptor, BLT1-expressing CHO cells, known to couple $\mathrm{G} \alpha_{\mathrm{I}}(35)$, increased impedance upon ligand addition (Supplemental Figure 3). This finding contrasts with LGR6-expressing CHO cells, where MaR1 reduced impedance (Figure 2). These results suggest that MaR1 initiates LGR6 coupling via a $G \alpha_{s}$ protein to stimulate cAMP. Also, using CyTOF and flow cytometry, MaR1 increased phosphorylation of CREB, the CAMP response element (CRE) binding protein in LGR6-dependent manner in THP-1 cells (Figure 6). Similar results were obtained with mouse peripheral blood monocytes and PMN (Figure 8). These results suggest that the MaR1-LGR6 axis could regulate CRE-containing genes (44) via cAMP and CREB.

MaR1 is present in human tissues identified via targeted MS/ MS/lipid mediator metabololipidomics (15-19) (reviewed in ref. 7). For example, human periodontal ligament stem cells biosynthesize SPMs, including MaR1 (45). MaR1 directly stimulates human $\mathrm{M} \Phi$ phagocytosis at $0.1-10 \mathrm{nM}$ and planarian tissue regeneration at 1-10 $\mathrm{nM}(10,12)$. MaR1-activated recombinant human LGR6 expressed in the chemiluminescent reporter cells as low as 0.01$10 \mathrm{nM}$, stimulated cAMP at $10 \mathrm{nM}$, impedance changes with $\mathrm{EC}_{50}$ approximately $1 \mathrm{nM}$, and wound repair at 1-10 $\mathrm{nM}$ (Figures 1, 2, and Supplemental Figure 4). These concentration ranges for recombinant LGR6-mediated MaR1 actions are comparable to those for MaR1 with primary human PMN and M (0.01-10 nM; Figures 4-6 and refs. 10,12). Thus, phagocyte LGR6 is likely to contribute to physiological proresolving functions of MaR1. In experimental systems, MaR1 exhibits potent actions in activating resolution of inflammation, tissue regeneration, organ protection, and resolving pain (7). The contribution and extent of MaR1-LGR6 interactions in each of these systems is of interest. In addition, MaR1 at nanomolar concentrations antagonizes activation of recombinant human BLT1 by the potent proinflammatory 
A

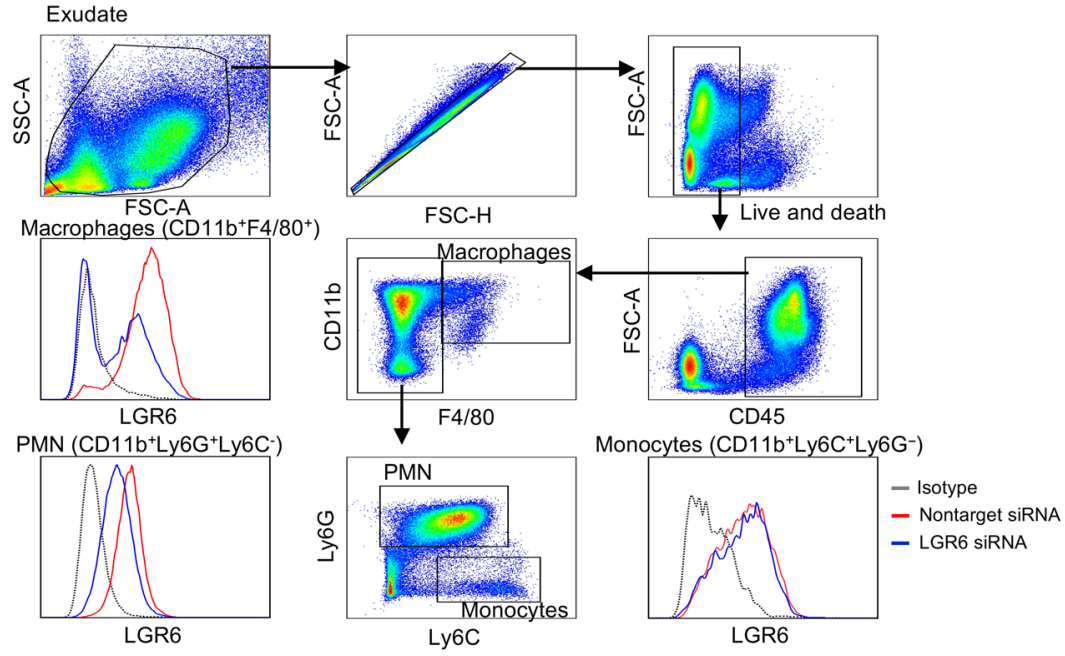

B

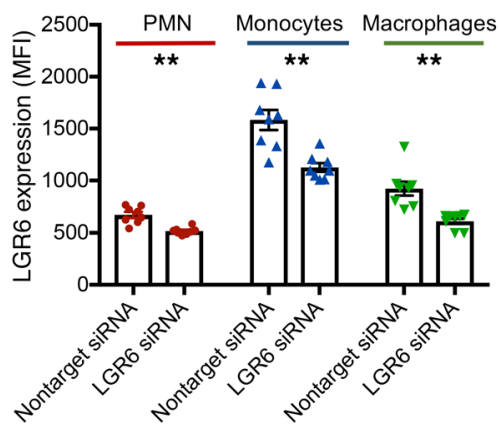

C

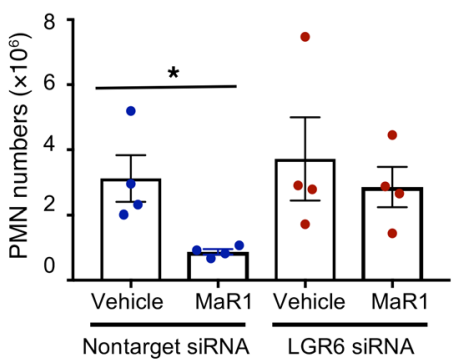

D

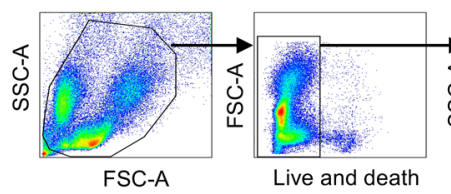

E

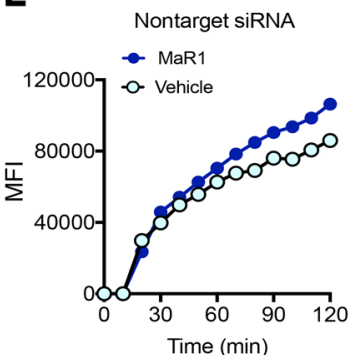

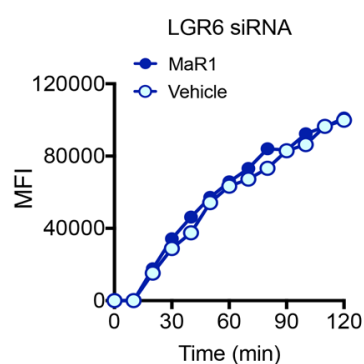

$\mathbf{F}$

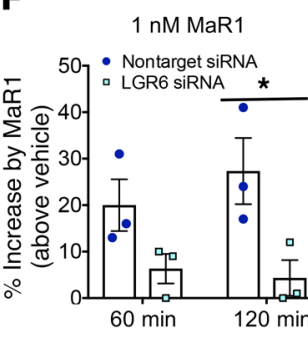

$$
\text { - Isotype }
$$
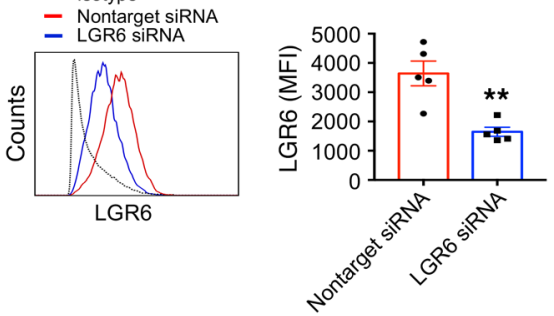

$10 \mathrm{nM}$ MaR1

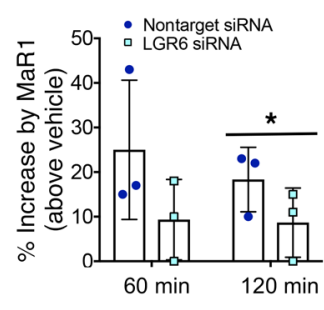

Figure 7. In vivo knockdown of mouse LGR6 reduces MaR1 actions in limiting PMN and stimulating macrophage phagocytosis. Mice were injected i.p. with siRNA for mouse LGR6 (10 $\mu \mathrm{g} /$ mouse) or control non-target siRNA. (A-C) Three days after siRNA injection, 1-mg zymosan was injected i.p. (time 0) to initiate peritonitis. At 12 hours, MaR1 (100 ng/mouse) was injected i.p., and peritoneal exudates were collected at 24 hours. (A) Flow cytometry gating strategy and histograms for LGR6 expression in specific leukocytes. Live cells were first selected from FSC and SSC dot plots, within which leukocytes (CD45+) were further selected to identify PMN (CD11b+Ly6C+Ly6C-), monocytes (CD11b+Ly6C-Ly6C+), and macrophages (CD11 b+F4/80+). (B) Quantification of LGR6 expression. ${ }^{* *} P<0.01$. LGR6 siRNA versus nontarget siRNA. Two-tailed unpaired Student's $t$ test. (C) Exudate PMN numbers. ${ }^{*} P<0.05$. Vehicle versus MaR1. Two-tailed unpaired Student's $t$ test. (D-F) Three days after siRNA injection, peritoneal macrophages were collected. (D) Flow cytometry gating strategy for macrophages (CD45+F4/80+Ly6C-), representative histograms, and quantification of LGR6 expression. ${ }^{* *} P<0.01$. LGR6 siRNA versus non-target siRNA. Two-tailed unpaired Student's $t$ test. (E) Phagocytosis of BacLight Green-labeled E. coli carried out using a real-time imaging microscope as in Figure 4. Results are MFI/cell from 4 fields/condition in 1 representative experiment with macrophages collected from nontarget siRNA (left) or LGR6 siRNA (right) injected mice. 1-nM MaR1. (F) Percent increases of phagocytosis by 1- or 10-nM MaR1 with macrophages collected from LGR6 siRNA or nontarget siRNA injected mice. Results are mean $\pm \operatorname{SEM}(n=3) .{ }^{*} P<0.05$. LGR6 siRNA versus nontarget siRNA. Two-tailed paired Student's $t$ test. 
mediator $\mathrm{LTB}_{4}$ (36). This MaR1-BLT1 interaction likely contributes to MaR1's anti-inflammatory and proresolving functions. Recently, MaR1 was demonstrated to activate retinoic acid-related orphan receptor $\alpha(\mathrm{ROR}-\alpha)$ in liver macrophages at nanomolar to micromolar concentrations, which in turn induces expression of 12-LOX and MaR1 biosynthesis. These results provide a MaR1/ ROR- $\alpha / 12-L O X$ circuit controlling nonalcoholic steatohepatitis $(46,47)$. These findings highlight the potential for MaR1 activation of nuclear receptors within the cells of origin, where intracellular concentrations of MaR1 may presumably reach nanomolar levels. Importantly, MaR1-stimulated phagocytosis is rapid and not dependent on ROR- $\alpha$. Thus, MaR1 exhibits cell-type specific and receptor-dependent actions. At picomolar to low nanomolar concentrations, as would be encountered in the extracellular milieu, for example, inflammatory exudates, MaR1 can activate LGR6, which transmits rapid signals to stimulate proresolving functions and signals. Together, we demonstrated that LGR6 is required for specific MaR1-dependent functions (phagocytosis) and/or intracellular signals (pCREB and pERK) with human MФ (Figure 4) and monocyte-like THP-1 (Figures 5 and 6) as well as with mouse MФ (Figure 7), PMN, and monocytes (Figure 8).

In summation, the present results provide evidence for MaR1 and LGR6 interactions in both human and mouse phagocytes, stimulating the main proresolving functions of phagocytes in resolution of inflammation. To substantiate the LGR6-dependent actions of MaR1, multiple lines of evidence were needed (Supplemental Figure 11). These include (a) MaR1 specific binding and activation of recombinant human LGR6 (e.g., $\beta$-arrestin recruitment, impedance change, cAMP increase), (b) MaR1-LGR6dependent stimulation of essential proresolving functions with human and mouse $\mathrm{M} \Phi$, namely, enhancing $\mathrm{M} \Phi$ phagocytosis and efferocytosis, and (c) MaR1-LGR6-dependent initiation of intracellular phosphorylation signals with human and mouse monocytes and PMN. This evidence indicates a new role of LGR6, in addition to its well-known role in adult stem cells and tissue regeneration. Taken together, our findings provide a new molecular means via MaR1-LGR6 to stimulate host resolution mechanisms to control prevalent diseases where excessive inflammation and/ or failed resolution are underlying culprits.

\section{Methods}

GPCR screening. A panel of 77 orphan GPCRs was screened using the PathHunter $\beta$-arrestin enzyme fragment complementation technology with $\beta$-galactosidase (DiscoverX). In this system, $\beta$-galactosidase was split into 2 inactive fragments - enzyme acceptor and enzyme donor. ProLink-tagged proteins were then generated: the enzyme acceptor was fused to $\beta$-arrestin and the enzyme donor was fused to the GPCRs in this panel. Activation of GPCR recruited $\beta$ arrestin to the receptor, bringing 2 inactive fragments to close proximity and restoring $\beta$-galactosidase activity, which was monitored via chemiluminescent signals using PathHunter detection reagents (DiscoverX). Custom GPCR screening was carried out with 10-nM MaR1 or vehicle control $(0.1 \%$ ethanol) using the agonist format. MaR1 was incubated with cells expressing the orphan panel of GPCRs at $37^{\circ} \mathrm{C}$ for 90 minutes. Negative controls measured potential constitutive activity in the absence of ligand. This custom screening was carried out in duplicate, and mean chemiluminescence was used for analysis as follows. For agonist format, the \% activity was calculated using the following formula: $\%$ activity $=100 \% \times($ mean RLU of test sample - mean RLU of vehicle control)/(mean RLU of vehicle control). RLU, relative luminescence units.

GPCR-beta-arrestin system. Ligand receptor interactions were monitored using the Beta Arrestin PathHunter eXpress system (DiscoverX) and carried out essentially as in ref. 48 , with CHO cells stably overexpressing recombinant human LGR6 or GPR148 receptors. Briefly, cells were plated in 96 -well plates 48 hours prior to experiments. Test compounds were incubated with cells for 1 hour at $37^{\circ} \mathrm{C}$, and receptor activation was determined by measuring chemiluminescence using the PathHunter detection kit (DiscoverX).

cAMP measurements. HEK-293 cells were transfected with a mock vector (pCMV6-AC-GFP; catalog no. PS100010) or with an expression plasmid for human LGR6 (NM_021636; GFP-tagged; catalog no. RG208537; Origene). $\mathrm{GFP}^{+}$cells were sorted using a BD FACSAria Cell Sorter and maintained in culture media DMEM supplemented with $10 \%$ FBS and G418 (1 mg/ml). The day before each experiment, $0.5 \times 10^{6}$ cells/well were plated onto 12 -well plates. The next day, media was aspirated and cells incubated with 50- $\mu \mathrm{M}$ IBMX in Dulbecco's Phosphate-Buffered Saline with $\mathrm{CaCl}_{2}$ and $\mathrm{MgCl}_{2}\left(\mathrm{DPBS}^{++}\right)$ for 10 minutes, followed by addition of test compounds for 15 minutes at $37^{\circ} \mathrm{C}$. Cells were frozen at $-80^{\circ} \mathrm{C}$ for 2 hours; cAMP was then measured following the manufacturer's instructions (Cyclic AMP Select ELISA kit, Cayman Chemicals).

Human leukocyte isolation and macrophage differentiation. Human peripheral blood was drawn from healthy volunteers, PMN, and monocytes were isolated (48). MФ were differentiated by culturing freshly isolated monocytes in RPMI media supplemented with $10 \%$ FBS and recombinant human GM-CSF (10 ng/ml, R\&D Systems) for M1, or M-CSF for M2 (20 ng/ml, R\&D Systems) for 7 days. Cells were then polarized to M1 with LPS $(100 \mathrm{ng} / \mathrm{ml})$ and IFN- $\gamma(20 \mathrm{ng} / \mathrm{ml})$ or M2 with IL-4 (20 ng/ml) (Peprotech) for 48 hours. For staining of M1 and M2 surface markers, human M1 and M2 macrophages were suspended in FACS buffer $\left(\mathrm{DPBS}^{++}\right.$with $1 \%$ bovine serum albumin and $0.1 \%$ sodium azide) and incubated with $\mathrm{FC}$ block ( 15 minutes, $4^{\circ} \mathrm{C}$; BD PharMingen). Macrophages were incubated with anti-human APC CD54 (clone HA58), anti-human FITC CD206 (clone 19.2) (BD Bioscience), anti-human PerCP/Cy5.5 CD163 (clone RM3/1) (Biolegend), and anti-human PE CD80 (clone 2D10) for phenotyping or CyTOF. All flow cytometric samples were assessed using FACSDiva Canto II (BD Biosciences) and analyzed using FlowJo version V10.0.8r1 (TreeStar).

Receptor expression. Human peripheral blood, MФ, THP1 (ATCC TIB-202), or HEK-293 (ATCC CRL-1573) cells were incubated with rabbit anti-human LGR6 (LS-A442) or rabbit nonimmune IgG (LSBio) for 30 minutes, followed by PE donkey anti-rabbit IgG (1:250) for 20 minutes. Murine peritoneal or whole blood leukocytes were incubated with rabbit anti-human/mouse LGR6 (17658-1-AP) or rabbit nonimmune IgG (Proteintech) followed by PE donkey anti-rabbit IgG (1:250) for 20 minutes. LGR6 expression was then assessed using flow cytometry.

Intracellular calcium release. Human peripheral leukocytes, M1, and M2 macrophages were resuspended in complete RPMI media (RPMI and 10\% FBS). Human peripheral leukocytes were labelled with surface markers APC Cy7-antihuman CD14 (monocytes), APC anti-human CD15 (neutrophils), and PerCP Cy5.5 anti-human CD3 (T lymphocytes) for 20 minutes. Macrophages were incubated with 
A

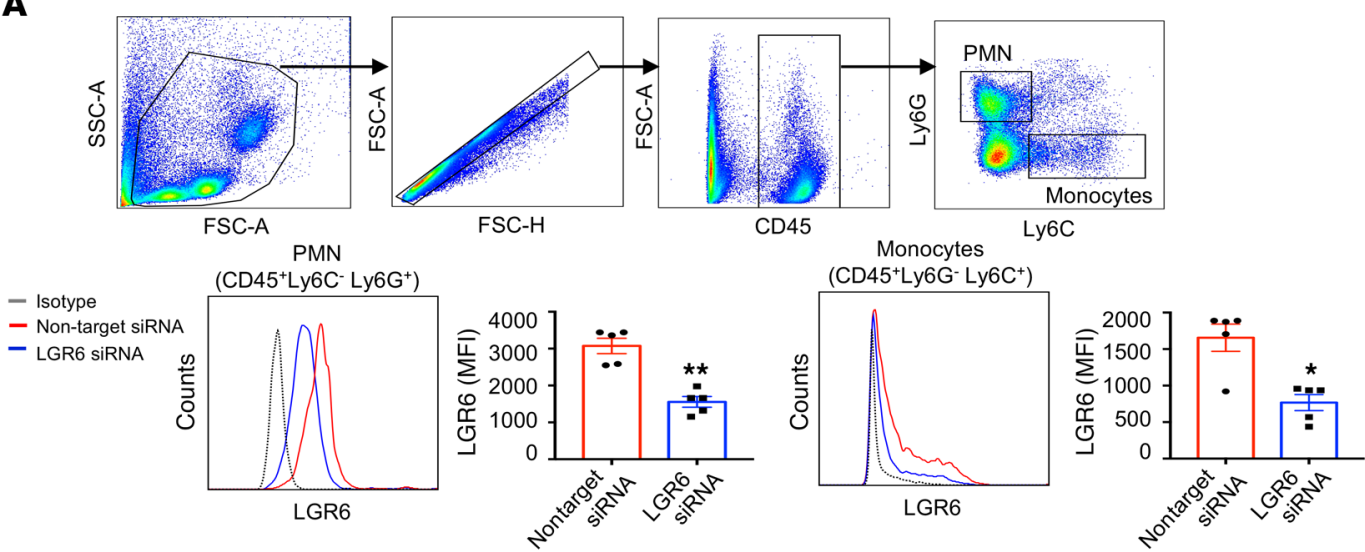

B

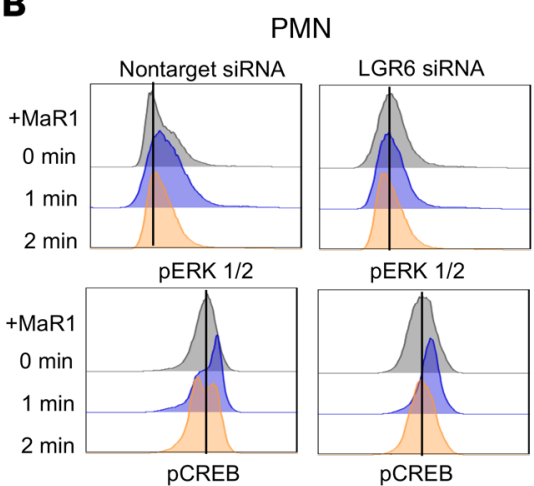

C

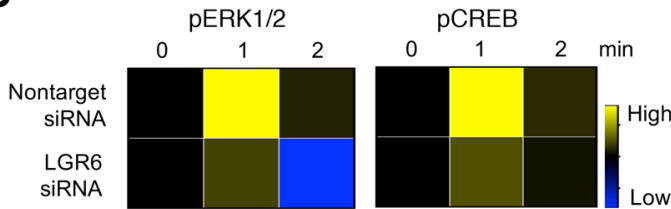

D

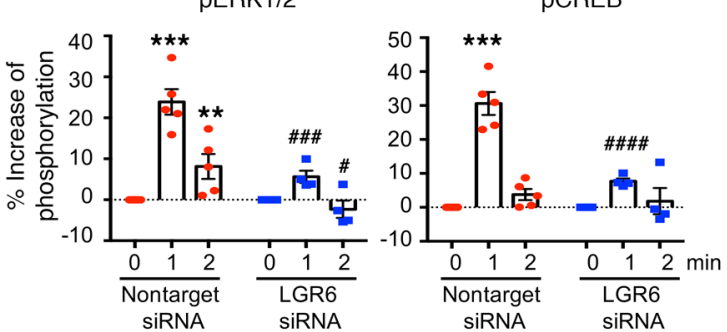

E
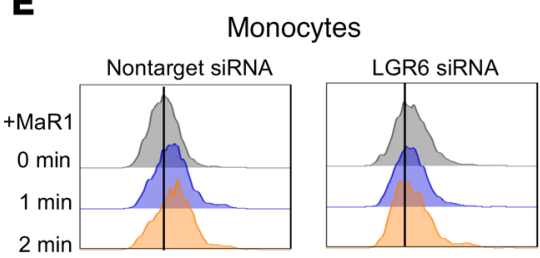

pERK $1 / 2$
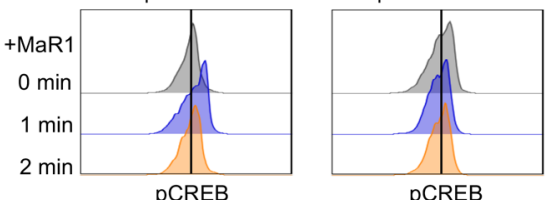

$\mathbf{F}$

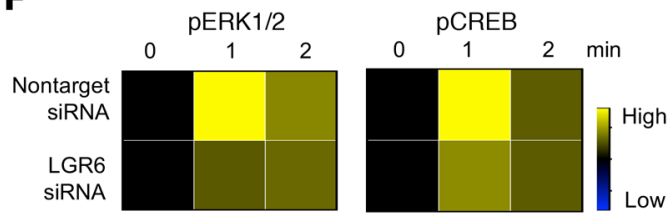

G

pERK $1 / 2$

PCREB
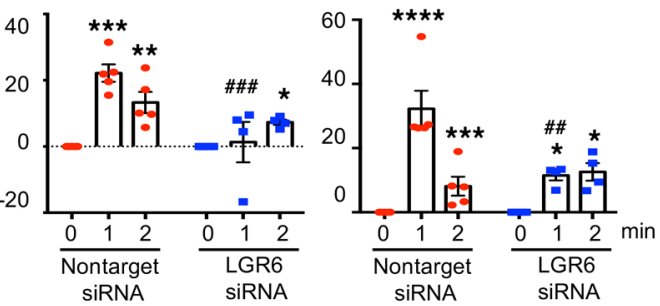

Figure 8. In vivo knockdown of mouse LGR6 diminishes MaR1 phosphorylation signals on peripheral blood PMN and monocytes. Mice were injected i.p. with siRNA for mouse LGR6 (10 $\mu \mathrm{g} /$ mouse) or control nontarget siRNA. Three days after siRNA injection, peripheral blood was collected and incubated with 10-nM MaR1 for 0 to 2 minutes. RBC was lysed and pCREB and pERK levels were determined using flow cytometry. (A) (Top panels) Flow cytometry gating strategy for PMN (CD11b+Ly6C+Ly6C-) and monocytes (CD11 b+Ly6C- Ly6C+). (Bottom panels) representative histograms and quantification of LGR6 expression. ${ }^{*} P<0.05$, ${ }^{* *} P<0.01$. LGR6 siRNA versus nontarget siRNA. Two-tailed unpaired Student's $t$ test. (B-D) $p C R E B$ and $p E R K$ levels in PMN.

(B) Representative histograms (C), heat maps, and (D) quantification. Results are mean \pm SEM $(n=5)$. (E-G) pCREB and $p E R K$ levels in monocytes. (E) Representative histograms (F), heat maps, and (G) quantification. Results are mean \pm SEM $(n=5)$. (D and $\mathbf{G}){ }^{*} P<0.05$; ${ }^{* *} P<0.01$; ${ }^{* * *} P<0.001$; ${ }^{* * *} P<$

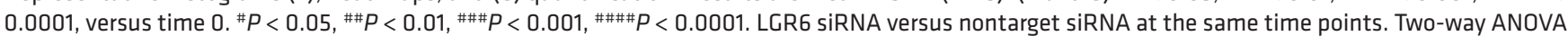
with Bonferroni's multiple comparisons test.

anti-human APC CD54 (clone HA58), anti-human FITC CD206 (clone 19.2) (BD Bioscience), anti-human PerCP/Cy5.5 CD163 (clone RM3/1) (Biolegend), and anti-human PE CD80 (clone 2D10). Cells were then washed and labeled with Indo-1 AM dye (ebioscience) at $37^{\circ} \mathrm{C}$ for 30 minutes. Samples were analyzed using BD FACSymphony (BD Biosciences) at approximately 2,000-2,500 events/ seconds for 5 minutes to establish the $\mathrm{Ca}^{2+}$ baseline with an Indo-1 AM signal, then $10 \mathrm{nM}$ of MaR1, $\mathrm{LTD}_{4}, \mathrm{LTB}_{4}$, or $1 \mu \mathrm{M}$ of Ionophore 
A23187 (positive control) was added to samples and recorded for another 10 minutes. All samples were analyzed using FlowJo.

ECIS. G protein coupling: Ligand-receptor interactions were determined by measuring impedance across CHO-K1 cell (ATCC CCL-61) monolayers using an ECIS (Applied Biophysics) (34), and carried out essentially as in ref. 48. Briefly, CHO cells were transfected with a mock vector (pCMV6-AC-GFP; catalog no. PS100010) or with an expression plasmid for human LGR6 (NM_021636; GFPtagged; catalog no. RG208537; Origene, Rockville, MD). $\mathrm{GFP}^{+}$cells were sorted using a BD FACSAria III Cell Sorter and maintained in culture media HAM F-12 supplemented with 10\% FBS and G418 $(1 \mathrm{mg} / \mathrm{ml})$. The day before each experiment, cells were plated onto 8-well ECIS chamber slides (8W10E+; $0.1 \times 10^{6} /$ well). The next day, test compounds were added to the chambers in HAM F-12 serum-free medium, and impedance changes were recorded every 4 seconds for 8 to 12 minutes at $37^{\circ} \mathrm{C}$.

Wound healing: Human epidermal fibroblasts (Lonza), LGR6or mock-transfected $\mathrm{CHO}$ cells were plated onto 8-well ECIS chamber slides (8W1E; $0.1 \times 10^{6}$ per well) $(38)$. The confluent cells were wounded $(1,250 \mu \mathrm{A}, 64 \mathrm{KHz}, 30 \mathrm{sec})$ and washed once. Fresh medium was added to cells in the presence of vehicle, MaR1 (1-100 nM) or $12 E$-MaR1 $(100 \mathrm{nM})$, and impedance changes were recorded every 10 minutes for 6 hours at $37^{\circ} \mathrm{C} .12 \mathrm{E}$-MaR1 was prepared earlier and qualified using NMR (12).

Preparation of $\left[{ }^{3} \mathrm{H}\right]-\mathrm{MaR} 1$ methyl ester and radioligand binding. Synthetic precursor 12,13-acetylenic MaR1 methyl ester was prepared by total organic synthesis (purchased as a custom order from Cayman Chemicals), and custom tritation was carried out by Dr. Gupta (American Radiolabeled Chemicals) by catalytic hydrogenation to give tritiated $\left[12,13-{ }^{3} \mathrm{H}\right]$ MaR1 methyl ester. The overall yield was low, precluding isolation of the free carboxylic acid of MaR1 and limiting the numbers of radiolabeled-MaR1 incubations. For each experiment, the integrity of the radioligand was confirmed and isolated using RP-UV-HPLC (1100 Series, Agilent Technologies) using an Poroshell 120 EC-18 column $(100 \mathrm{~mm} \times 4.6 \mathrm{~mm} \times 2.7 \mu \mathrm{m}$; Agilent Technologies) coupled with a DAD (G1315B, Agilent Technologies). A gradient of methanol/water of 55:45 (v/v) was ramped to 63:37 (v/v) over 22 minutes and then to $98: 2(\mathrm{v} / \mathrm{v})$ for the next 8 minutes. The flow rate was maintained at $0.5 \mathrm{ml} / \mathrm{min}$.

$\left[{ }^{3} \mathrm{H}\right]$-MaR1 methyl ester binding was performed with $\mathrm{CHO}$ cells transfected with LGR6. Cells were suspended in $\mathrm{DPBS}^{++}$. For competition binding, cells $\left(0.5 \times 10^{6}\right.$ cells $\left./ 0.1 \mathrm{ml}\right)$ were incubated with approximately $2 \mathrm{nM}$ of $\left[{ }^{3} \mathrm{H}\right]$-MaR1 methyl ester in the absence or presence of increasing concentrations of unlabeled MaR1 or related compounds for 60 minutes at $4^{\circ} \mathrm{C}$. The bound and unbound radioligands were separated by filtration through Whatman GF/C glass microfiber filters (Fisher). Filters were washed twice with 5-ml ice-cold DPBS. The radioactivity retained on the filter was determined using a scintillation counter (Beckman). Non-specific binding was determined in the presence of $10 \mu \mathrm{M}$ of unlabeled homoligands.

PMN chemotaxis. Peripheral blood neutrophils were isolated by density gradient and suspended in DPBS (Lonza). For real-time monitoring using ibidi chambers, PMN $\left(3 \times 10^{6}\right.$ cells $\left./ \mathrm{ml}\right)$ were placed at ibidi chemotaxis chambers and the manufacturer's instructions were followed ( $\mu$-Slide Chemotaxis 80326, ibidi cells in focus). PMN were incubated with vehicle $\left(\mathrm{DPBS}^{++}\right.$plus $0.1 \%$ ethanol) or MaR1 (1 or 10 $\mathrm{nM})$ for 10 minutes at $37^{\circ} \mathrm{C}$. Compounds were prepared in $\mathrm{DPBS}^{++}$.
Chemotaxis toward $10 \mathrm{nM}$ of IL-8 was then recorded over 2 hours with a digital monochrome/color switching camera using BZ-II Viewer software (Keyence). Cell migration was analyzed using the cell-tracking function, and tracks were analyzed utilizing Chemotaxis and the Migration Tool in ImageJ software. Twenty cells per field, and 3 fields per condition were analyzed. For Neuro Probe ChemoTx System plates (5- $\mu \mathrm{m}$ pore size filter; Neuro Probe), $10-\mathrm{nM}$ IL- 8 was added to the bottom chamber. PMN labelled with carboxyfluorescein succinimidyl ester were incubated with mouse anti-human LGR IgG1, rabbit anti-human LGR6 IgG, or isotype controls for 30 minutes at room temperature, followed by addition of MaR1 $(0.1 \mathrm{nM}-100 \mathrm{nM})$ or vehicle for 15 minutes. Cells were then added onto the top of filters to initiate chemotaxis toward IL-8 for 2 hours $\left(37^{\circ} \mathrm{C}\right.$ in $\left.5 \% \mathrm{CO}_{2}\right)$. Migrated cells on the bottom chambers were quantified using fluorescence measured on a SpectraMax M3 plate reader (Molecular Devices Inc.).

LGR6 overexpression and macrophage phagocytosis. For overexpression of LGR6, MФ $\left(5 \times 10^{6}\right.$ cells in a $10-\mathrm{cm}$ Petri dish $)$ were transfected with a mock vector (pCMV6-Entry; catalog no. PS100001) or with expression plasmids for human LGR6 (NM_021636; untagged; catalog no. SC304949; Origene) using Jet-Pei transfection reagent following the manufacturer's instructions (Polyplus-transfection SA). For knockdown of LGR6, MФ $\left(5 \times 10^{6}\right.$ cells in a 10-cm Petri dish) were transfected with LGR6 shRNA (catalog no. TR311746, Origene) or scramble-control shRNA. For real-time imaging, 48 hours after transfection, $\mathrm{M} \Phi$ were plated onto 8-well chamber slides $\left(0.1 \times 10^{6}\right.$ cells/well in $\mathrm{DPBS}^{++}$). Twenty-four hours later, imaging was carried out. Chamber slides were kept in a Stage Top Incubation system for microscopes equipped with a built-in digital gas mixer and temperature regulator (TOKAI HIT model INUF-K14). MaR1 was added to MФ (1-10 nM, $15 \mathrm{~min})$ followed by BacLight Green-labeled E. coli $\left(5 \times 10^{6}\right.$ CFUs). Images were then acquired every 10 minutes for 3 hours $\left(37^{\circ} \mathrm{C}\right)$ with a Keyence BZ-9000 (BIOREVO) inverted fluorescence phasecontrast microscope (20X objective) equipped with a monochrome/ color switching camera using BZ-II Viewer software. Green fluorescence intensity was quantified using the BZ-II Analyzer. For doseresponse studies, $\mathrm{M} \Phi$ were plated onto 96-well plates (50,000 cells/ well in $\left.\mathrm{DPBS}^{++}\right)$, and phagocytosis was carried out 24 hours later. MaR1 (0.1 pM-10 nM) was incubated with $\mathrm{M} \Phi$ for 15 minutes at $37^{\circ} \mathrm{C}$, followed by incubation with FITC-labeled zymosan particles at a 10:1 ratio (zymosan: MФ), CFDA-labeled apoptotic PMN at 5:1 ratios, or fluorescent-labeled E. coli (BacLight Green, Molecular Probes) at a 50:1 ratio for 60 minutes at $37^{\circ} \mathrm{C}$. Plates were gently washed, extracellular fluorescence quenched by trypan blue, and phagocytosis determined by measuring total fluorescence (Ex 493/Em $535 \mathrm{~nm}$ ) using SpectraMax M3 plate reader (Molecular Probes).

Lipid mediator metabololipidomics. Liquid chromatography-mass spectrometry-based metabololipidomics were performed with human macrophages. Prior to sample extraction, ice-cold methanol containing deuterium-labeled $\mathrm{d}_{4}-\mathrm{LTB}_{4}, \mathrm{~d}_{4}-5$ S-HETE, $\mathrm{d}_{4}-\mathrm{PGE}_{2}, \mathrm{~d}_{5}$-RvD2, and $\mathrm{d}_{5}-$ $\mathrm{LXA}_{4}$ internal standards (500 pg each) were added to facilitate quantification. All samples were kept at $-20^{\circ} \mathrm{C}$ for 45 minutes to allow protein precipitation and then subjected to solid-phase extraction as described (10-12). Extracted samples were analyzed by a liquid chromatography-ultraviolet-tandem mass spectrometry system, QTrap 5500 (AB Sciex) equipped with a Shimadzu LC-20AD HPLC (Tokyo, Japan). A 120 EC-18 column (100 mm $\times 4.6 \mathrm{~mm} \times 2.7 \mu \mathrm{m}$; Agilent Technologies $)$ was kept in a column oven maintained at $50^{\circ} \mathrm{C}$, and LMs were eluted 
with a gradient of methanol/water/acetic acid from 55:45:0.01 (v/v/v) to 100:0:0.01 at $0.5 \mathrm{ml} / \mathrm{min}$ flow rate. To monitor and quantify the levels of targeted LM, a multiple reaction monitoring method was devised with signature ion fragments for each molecule. Identification was conducted using published criteria including retention times and at least 6 diagnostic ions. Calibration curves were obtained using synthetic and authentic LM mixtures, including $\mathrm{d}_{4}-\mathrm{LTB}_{4}, \mathrm{~d}_{5}-\mathrm{LXA}_{4}, \mathrm{~d}_{4}-\mathrm{PGE}_{2}, \mathrm{~d}_{5}$ RvD2, RvD1, RvD2, RvD5, PD1, MaR1, RvE1, RvE2, LXA 4 , LXB 4 , PGE 2 , $\mathrm{PGD}_{2}, \mathrm{PGF}_{2 \alpha}, \mathrm{TXB}_{2}, \mathrm{LTB}_{4}$ at $1.56,3.12,6.25,12.5,25,50,100 \mathrm{pg}$. Linear calibration curves for each compound were obtained with $\mathrm{r}^{2}$ values of 0.98-0.99. Quantification was carried out based on peak areas of the multiple reaction monitoring transitions.

LGR6 knockdown, THP-1 phagocytosis, and signaling. Stable THP-1 and HEK-293 shRNA GFP reporter cell lines were generated by using a lentivirus GFP-based shRNA expression vector miRE-SGEP, a gift from C. Fellmann (49). Briefly, the top 3 human LGR6 de novo prediction shRNA sequences were obtained by using SplashRNA algorithm (50). LGR6 shRNA sequences (Supplemental Table 4) and Renilla Firefly Luciferase shRNA (Ren.713) sequences (as negative control) were cloned into miRE-SGEP and transduced into either HEK or THP-1 cells as described by Fellmann et al. (49). Transduction efficiency of miRE-SGEP lentivirus was measured after 48 hours by flow cytometry and cells were then selected with puromycin $(2.5 \mu \mathrm{g} / \mathrm{ml})$. GFP ${ }^{+}$THP-1 (>90\%) were then sorted for establishing the stable THP-1 cell lines. LGR6 gene knockdown was confirmed by surface expression of LGR6 by flow cytometry.

For phagocytosis, shRNA LGR6 (shRNA LGR6.SGEP), shRNA Ren713 (Ren.713.SGEP), or mock vector (SGEP alone) transfected THP-1 cells were treated with 10-nM MaR1 or vehicle (0.01\% ethanol) 15 minutes prior to adding BacLight Red-labeled (PE-Texas Red) E. coli (1:50 THP-1:E.coli) for 45 minutes at $37^{\circ} \mathrm{C}$. Cells were fixed with $4 \%$ paraformaldehyde for 15 minutes and then analyzed by flow cytometry to assess phagocytosis by determining PE-Texas Red intensities in both $\mathrm{GFP}^{+}$and GFP- populations.

For intracellular signaling, shRNA LGR6 (shRNA LGR6.SGEP) or mock vector (SGEP alone) THP-1 cells were incubated with $10-\mathrm{nM}$ MaR1 for $0,1,2$, and 5 minutes and at $37^{\circ} \mathrm{C}$, followed by $1.6 \%$ of paraformaldehyde for 10 minutes at room temperature. Cells were permeabilized in $80 \%$ ice-cold methanol for 10 minutes at $-20^{\circ} \mathrm{C}$, washed twice, and then stained with PE-pERK1/2 (T202/Y204) (clone MILAN8R) (ebioscience, San Diego) and APC-pCREB (S133) (clone 4D11) (Invitrogen, Carlsbad, California) or respective Isotype controls. Levels of pERK1/2 and pCREB were then assessed using flow cytometry.

CyTOF. Human M1 and M2 macrophages were prepared and incubated with 10-nM MaR1 for $0,1,2,5$, and 15 minutes at $37^{\circ} \mathrm{C}$, followed by $1.6 \%$ of paraformaldehyde for 10 minutes at room temperature. Cells were barcoded following the manufacturer's protocol with palladium isotopes (Pd 102, 104, 105, 108, and 110) (Fluidigm, Science). Briefly, cells were washed twice using barcoding permeabilization buffer. Diluted barcodes were transferred to cells and incubated for 30 minutes at room temperature. Barcoded cells were washed twice in CyTOF staining buffer $\left(\mathrm{DPBS}^{++}\right.$with $0.5 \%$ BSA and $0.1 \%$ sodium azide) and then pooled for staining. Pooled barcoded cells were incubated for 10 minutes with FcX block (Biolegend) for Fc receptor-mediated nonspecific antibody binding. Cells were stained for 30 minutes with metal-label surface antibodies at room temperature, then washed twice in CyTOF staining buffer. Cells were then permeabilized in
$80 \%$ ice-cold methanol for 10 minutes at $-20^{\circ} \mathrm{C}$. After washing twice to remove the methanol, macrophages were stained with metal-conjugated antibodies for intracellular phosphoproteins at room temperature for 30 minutes. The antibodies used for CyTOF are listed in Supplemental Table 5. Cells were washed twice and stained in $500 \mu \mathrm{l}$ of 1:1,000 Iridium intercalator (DVS Science, Toronto) diluted in DPBS overnight at $4^{\circ} \mathrm{C}$. Cells were then washed twice in CyTOF staining buffer, twice in MilliQ-filtered deionized water, then reconstituted at a concentration of $5 \times 10^{6}$ cells $/ \mathrm{ml}$ containing EQ calibration beads (EQ four elements Calibration Beads, Fluidigm, Science) according to the manufacturer's protocol. Barcoded cells were analyzed on Helios CyTOF (Fluidigm, Science) at an event rate of 400 to 500 cells per second. The data were normalized using v6.3.119 Helios Software (Fluidigm) at LMA CyTOF facility at Dana Farber Cancer Institute (Boston, Massachusetts, USA). Files were debarcoded using Fluidigm Debarcoder application. Gating was performed in Cytobank Platform (Cytobank). Phosphorylation levels were calculated as the difference between the inverse hyperbolic sine (arcsinh) of the median signal intensity at indicated time points and the arcsinh of the median signal intensity in unstimulated (0 min) signal (51).

Murine zymosan-initiated peritonitis: in vivo knockdown of LGR6. Mice were anesthetized with isoflurane and experiments carried out with male FVB mice (6-8 weeks; Jackson Laboratory; lab diet containing essential fatty acids from supplier). For LGR6 knockdown, mice were injected i.p. with siRNA for mouse LGR6 (10 $\mu$ g; catalog no. E-04405600-0010, Dharmacon Accell siRNA pool) or nontarget siRNA (10 $\mu \mathrm{g}$; nontargeting siRNA no. 1; catalog no. D-001910-01-05). Three days later, zymosan (1 mg, i.p.) was injected to initiate peritonitis. Twelve hours later, MaR1 (100 ng, i.p.) was given and inflammatory exudates were collected at 24 hours. Total leukocyte phenotyping was assessed by flow cytometry. Murine peritoneal exudates were incubated with live/death Fixable Aqua Dead stain (ThermoFisher), anti-mouse PerCP/Cy5.5 CD45 (clone 30-F11 Biolegend), anti-mouse PE/Cy7 CD11b (clone M1/70, eBioscience), anti-mouse APC F4/80 (clone BM8, eBioscience), anti-mouse FITC Ly6C (clone HK1.4, Biolegend), and anti-mouse PE Ly6G (clone 1A8, Biolegend) or appropriate isotype controls. Peritoneal exudates populations were gated on live cells, then

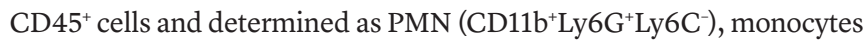
$\left(\mathrm{CD} 11 \mathrm{~b}^{+}\right.$Ly6G-Ly6C $\left.{ }^{+}\right)$, and macrophages $\left(\mathrm{CD} 11 \mathrm{~b}^{+} \mathrm{F} 4 / 80^{+}\right)$.

In separate sets of experiments, 3 days after siRNA injection, peritoneal MФ and peripheral blood were collected. Peritoneal MФ was used to assess MaR1-stimulated phagocytosis. МФ were plated onto 8-well chamber slides $\left(0.1 \times 10^{6}\right.$ cells/well in $\left.\mathrm{DPBS}^{++}\right)$, and phagocytosis of BacLight Green-labeled E. coli was carried out (see previous information). Peripheral blood was collected from mice given either nontarget siRNA or LGR6 siRNA, followed by red blood cell lysis. Cells $\left(2 \times 10^{6}\right.$ cells $)$ were incubated with $10-\mathrm{nM} \mathrm{MaR} 1$ for 0,1 , and 2 minutes and at $37^{\circ} \mathrm{C}$, followed by addition of $1.6 \%$ of paraformaldehyde for 10 minutes at room temperature. Cells were stained with anti-mouse PerCP/Cy5.5 CD45, anti-mouse FITC Ly6C, and anti-mouse PE Ly6G or appropriate isotype controls. Cells were then permeabilized in $80 \%$ ice-cold methanol for 10 minutes at $-20^{\circ} \mathrm{C}$. Cells were washed twice, then stained for intracellular with PE-pERK1/2 (T202/Y204) and APC-pCREB (S133) or respective isotype controls. Flow cytometry was carried out using FACSDiva Canto II or LSRFortessa (BD Biosciences) and analyzed using FlowJo.

Statistics. Statistical analyses were performed using 2-tailed Student's $t$ test for 2 group comparisons, 1-way ANOVA with multiple group 
comparisons for 3 or more independent groups, or 2-way ANOVA with multiple group comparisons for 2 biological variables (GraphPad Prism). $P$ values of less than 0.05 were taken to be significant.

Study approval. Mice (male FVB mice, 6-8 weeks) were used in this study in accordance with the Standing Committee on Animals of Brigham and Women's Hospital (protocol no. 2016N000145). Human peripheral blood was drawn from healthy volunteers by venipuncture using a heparinized syringe (Partners Human Research Committee Protocol no. 1999-P-001297). The written informed consent from each participant who denied taking medications at least 2 weeks prior to donation was received.

\section{Author contributions}

$\mathrm{NC}$ designed and conducted experiments, acquired and analyzed data, and wrote the manuscript. SL designed and conducted experiments and acquired and analyzed data. PCN and XR conducted experiments and acquired and analyzed data. CNS conceived the overall research, designed experiments, and wrote the manuscript.

\section{Acknowledgments}

The authors thank Mary Small for assistance with manuscript preparation, Christof Fellmann (University of California, Berkeley) for expert help with shRNA design, and Johannes Zuber (Research Institute of Molecular Pathology, Vienna, Austria) for the gift of shRNA constructs. This work was supported by NIH grant R01GM038765 (to CNS) and R01GM038765-29S1 (to SL).

Address correspondence to: Charles N. Serhan, Center for Experimental Therapeutics and Reperfusion Injury, Hale Building for Transformative Medicine 3-016, 60 Fenwood Road, Boston, Massachusetts 02115, USA. Phone: 617.525.5001; Email: cserhan@ bwh.harvard.edu.
1. Majno G, Joris I. Cells, Tissues, and Disease: Principles of general pathology. New York, New York, USA: Oxford University Press; 2004.

2. Serhan CN, Savill J. Resolution of inflammation: the beginning programs the end. Nat Immunol. 2005;6(12):1191-1197.

3. Mantovani A, Cassatella MA, Costantini C, Jaillon S. Neutrophils in the activation and regulation of innate and adaptive immunity. Nat Rev Immunol. 2011;11(8):519-531.

4. Delano MJ, Ward PA. The immune system's role in sepsis progression, resolution, and long-term outcome. Immunol Rev. 2016;274(1):330-353.

5. Levine S, Malone E, Lekiachvili A, Briss P. Health care industry insights: why the use of preventive services is still low. Prev Chronic Dis. 2019;16:E30.

6. Perretti M, Cooper D, Dalli J, Norling LV. Immune resolution mechanisms in inflammatory arthritis. Nat Rev Rheumatol. 2017;13(2):87-99.

7. Serhan CN, Chiang N, Dalli J. New pro-resolving n-3 mediators bridge resolution of infectious inflammation to tissue regeneration. Mol Aspects Med. 2018;64:1-17.

8. Wallace JL, Ianaro A, Flannigan KL, Cirino G. Gaseous mediators in resolution of inflammation. Semin Immunol. 2015;27(3):227-233.

9. Fredman G. DELineating resolution of inflammation. Nat Immunol. 2019;20(1):2-3.

10. Serhan CN, et al. Maresins: novel macrophage mediators with potent antiinflammatory and proresolving actions. J Exp Med. 2009;206(1):15-23.

11. Deng B, et al. Maresin biosynthesis and identification of maresin 2, a new anti-inflammatory and pro-resolving mediator from human macrophages. PLoS One. 2014;9(7):e102362.

12. Serhan CN, et al. Macrophage proresolving mediator maresin 1 stimulates tissue regeneration and controls pain. FASEB J. 2012;26(4):1755-1765.

13. Rodriguez AR, Spur BW. Total synthesis of the macrophage derived anti-inflammatory lipid mediator Maresin 1. Tetrahedron Lett. 2012;53(32):4169-4172.

14. Serhan CN, Dalli J, Colas RA, Winkler JW, Chiang N. Protectins and maresins: new pro-resolving families of mediators in acute inflammation and resolution bioactive metabolome. Biochim Biophys Acta. 2015;1851(4):397-413.

15. Barden AE, Moghaddami M, Mas E, Phillips M, Cleland LG, Mori TA. Specialised pro-resolving mediators of inflammation in inflammatory arthritis. Prostaglandins Leukot Essent Fatty Acids. 2016;107:24-29.

16. Clària J, Nguyen BT, Madenci AL, Ozaki CK, Serhan CN. Diversity of lipid mediators in human adipose tissue depots. Am J Physiol Cell Physiol. 2013;304(12):C1141-C1149.

17. Markworth JF, et al. Divergent shifts in lipid mediator profile following supplementation with n-3 docosapentaenoic acid and eicosapentaenoic acid. FASEB J. 2016;30(11):3714-3725.

18. Motwani MP, et al. Pro-resolving mediators promote resolution in a human skin model of UV-killed Escherichia coli-driven acute inflammation. JCI Insight. 2018;3(6):94463.

19. Zhu M, et al. Pro-resolving lipid mediators improve neuronal survival and increase $\mathrm{A} \beta 42$ phagocytosis. Mol Neurobiol. 2016;53(4):2733-2749.

20. Francos-Quijorna I, et al. Maresin 1 promotes inflammatory resolution, neuroprotection, and functional neurological recovery after spinal cord injury. J Neurosci. 2017;37(48):11731-11743.

21. Yang $\mathrm{T}$, et al. Maresin 1 attenuates neuroinflammation in a mouse model of perioperative neurocognitive disorders. Br JAnaesth. 2019;122(3):350-360.

22. Nordgren TM, et al. Maresin-1 reduces airway inflammation associated with acute and repetitive exposures to organic dust. Transl Res. 2015;166(1):57-69.

23. Snippert HJ, et al. Lgr6 marks stem cells in the hair follicle that generate all cell lineages of the skin. Science. 2010;327(5971):1385-1389.

24. Shimizu T. Lipid mediators in health and disease: enzymes and receptors as therapeutic targets for the regulation of immunity and inflammation. Annu Rev Pharmacol Toxicol. 2009;49:123-150.

25. Pierce KL, Premont RT, Lefkowitz RJ. Seventransmembrane receptors. Nat Rev Mol Cell Biol. 2002;3(9):639-650.

26. de Lau W, et al. Lgr5 homologues associate with Wnt receptors and mediate R-spondin signalling. Nature. 2011;476(7360):293-297.
27. Gong X, Carmon KS, Lin Q, Thomas A, Yi J, Liu Q. LGR6 is a high affinity receptor of R-spondins and potentially functions as a tumor suppressor. PLoS One. 2012;7(5):e37137.

28. Bystrom J, et al. Resolution-phase macrophages possess a unique inflammatory phenotype that is controlled by cAMP. Blood. 2008;112(10):4117-4127.

29. Wan $M$, et al. Prostaglandin $\mathrm{E}_{2}$ suppresses hCAP18/LL-37 expression in human macrophages via EP2/EP4: implications for treatment of Mycobacterium tuberculosis infection. FASEB J. 2018;32(5):2827-2840.

30. Woodward DF, Jones RL, Narumiya S. International Union of Basic and Clinical Pharmacology. LXXXIII: classification of prostanoid receptors, updating 15 years of progress. Pharmacol Rev. 2011;63(3):471-538.

31. Chiang N, Serhan CN. Structural elucidation and physiologic functions of specialized proresolving mediators and their receptors. $\mathrm{Mol}$ Aspects Med. 2017;58:114-129.

32. Bang S, Xie YK, Zhang ZJ, Wang Z, Xu ZZ, Ji RR. GPR37 regulates macrophage phagocytosis and resolution of inflammatory pain. J Clin Invest. 2018;128(8):3568-3582.

33. Sica A, Mantovani A. Macrophage plasticity and polarization: in vivo veritas. J Clin Invest. 2012;122(3):787-795.

34. Peters MF, Scott CW. Evaluating cellular impedance assays for detection of GPCR pleiotropic signaling and functional selectivity. J Biomol Screen. 2009;14(3):246-255.

35. Yokomizo T, Izumi T, Chang K, Takuwa Y, Shimizu T. A G-protein-coupled receptor for leukotriene B4 that mediates chemotaxis. Nature. 1997;387(6633):620-624.

36. Colas RA, et al. Identification and actions of the maresin 1 metabolome in infectious inflammation. J Immunol. 2016;197(11):4444-4452.

37. Lehoczky JA, Tabin CJ. Lgr6 marks nail stem cells and is required for digit tip regeneration. Proc Natl Acad Sci U S A . 2015;112(43):13249-13254.

38. Keese CR, Wegener J, Walker SR, Giaever I. Electrical wound-healing assay for cells in vitro. Proc Natl Acad Sci U S A. 2004;101(6):1554-1559. 39. Norris PC, Libreros S, Chiang N, Serhan CN. A 
cluster of immunoresolvents links coagulation to innate host defense in human blood. Sci Signal. 2017;10(490):eaan1471.

40. Hsu SY, et al. The three subfamilies of leucinerich repeat-containing $G$ protein-coupled receptors (LGR): identification of LGR6 and LGR7 and the signaling mechanism for LGR7. Mol Endocrinol. 2000;14(8):1257-1271.

41. Chiang N, Riley IR, Dalli J, Rodriguez AR, Spur $\mathrm{BW}$, Serhan CN. New maresin conjugates in tissue regeneration pathway counters leukotriene $\mathrm{D}_{4}$-stimulated vascular responses. FASEB J. 2018;32(7):4043-4052.

42. Ren W, et al. Single Lgr5- or Lgr6-expressing taste stem/progenitor cells generate taste bud cells ex vivo. Proc Natl Acad Sci U S A. 2014;111(46):16401-16406.
43. Szenker-Ravi E, et al. RSPO2 inhibition of RNF43 and ZNRF3 governs limb development independently of LGR4/5/6. Nature. 2018;557(7706):564-569.

44. Wen AY, Sakamoto KM, Miller LS. The role of the transcription factor CREB in immune function. JImmunol. 2010;185(11):6413-6419.

45. Cianci E, et al. Human periodontal stem cells release specialized proresolving mediators and carry immunomodulatory and prohealing properties regulated by lipoxins. Stem Cells Transl Med. 2016;5(1):20-32.

46. Han YH, et al. A maresin 1/ROR $\alpha / 12$-lipoxygenase autoregulatory circuit prevents inflammation and progression of nonalcoholic steatohepatitis. JClin Invest. 2019;130:1684-1698.

47. Spite M. Resolving inflammation in nonalcoholic steatohepatitis. JClin Invest. 2019;130:1524-1526.

48. Krishnamoorthy S, et al. Resolvin D1 binds human phagocytes with evidence for proresolving receptors. Proc Natl Acad Sci U S A. 2010;107(4):1660-1665.

49. Fellmann C, et al. An optimized microRNA backbone for effective single-copy RNAi. Cell Rep. 2013;5(6):1704-1713.

50. Pelossof R, et al. Prediction of potent shRNAs with a sequential classification algorithm. Nat Biotechnol. 2017;35(4):350-353.

51. Mingueneau M, et al. Single-cell mass cytometry of TCR signaling: amplification of small initial differences results in low ERK activation in NOD mice. Proc Natl Acad Sci U S A. 2014;111(46):16466-16471. 